\title{
LOCALLY COMPACT VECTOR SPACES AND ALGEBRAS OVER DISCRETE FIELDS $\left({ }^{1}\right)$
}

\author{
BY \\ SETH WARNER
}

The structure of locally compact vector spaces over complete division rings topologized by a proper absolute value (or, more generally, over complete division rings of type $V$ ) is summarized in the now classical theorem that such spaces are necessarily finite-dimensional and are topologically isomorphic to the cartesian product of a finite number of copies of the scalar division ring [9, Theorems 5 and 7], [6, pp. 27-31].

Here we shall continue a study of locally compact vector spaces and algebras over infinite discrete fields that was initiated in [16]. Since any topological vector space over a topological division ring $K$ remains a topological vector space if $K$ is retopologized with the discrete topology, some restriction needs to be imposed in our investigation, and the most natural restriction is straightness: A topological vector space $E$ over a topological division ring $K$ is straight if for every nonzero $a \in E, f_{a}: \lambda \rightarrow \lambda a$ is a homeomorphism from $K$ onto the one-dimensional subspace generated by $a$. Thus if $K$ is discrete, a straight $K$-vector space is one all of whose one-dimensional subspaces are discrete. Any Hausdorff vector space over a division ring topologized by a proper absolute value is straight [6, Proposition 2 , p. 25].

In $\S 1$ we shall prove that if $E$ is an indiscrete straight locally compact vector space over an infinite discrete division ring $K$, then $K$ is an absolutely algebraic field of prime characteristic and $[E: K]>\boldsymbol{\aleph}_{0}$. In $\S 2$ we shall see that finitedimensional subspaces of straight locally compact vector spaces need not be discrete. In $\S 3$ we shall see that the validity of the Open Mapping and Closed Graph theorems for straight locally compact vector spaces depends on whether those spaces are generated by compact subsets. In $\S 4$ we shall study locally compact algebras of linear operators. In $\$ 5$ we shall obtain structure theorems for a commutative semisimple straight locally compact algebra over an infinite discrete field; such an algebra is the topological direct product of a discrete algebra and a local subdirect product of a sequence of algebras, each discrete and algebraically the cartesian product of a family of fields, relative to finite subfields.

1. Straight locally compact vector spaces over infinite discrete fields. First we shall determine which infinite discrete division rings admit indiscrete straight

Received by the editors November 24, 1966.

( $\left.{ }^{1}\right)$ Research supported by National Science Foundation Grant GP-4473. 
locally compact vector spaces. We recall that an absolutely algebraic field is a field algebraic over its prime subfield.

Thus the absolutely algebraic fields of prime characteristic are the subfields of the algebraic closures of the prime fields $Z_{p}, p$ is a prime; such fields are necessarily countable.

THEOREM 1. If $K$ is an infinite discrete division ring, then there exists an indiscrete straight locally compact vector space over $K$ if and only if $K$ is an absolutely algebraic field of prime characteristic.

Proof. In [16, Example 1] the existence of an indiscrete straight locally compact vector space over a discrete infinite absolutely algebraic field of prime characteristic was shown. Conversely, let $E$ be an indiscrete straight locally compact vector space over an infinite discrete division ring $K$. By [16, Theorem 5], the characteristic of $K$ is a prime. Let $P$ be the prime subfield of $K$, let $\alpha \in K$, and let $V$ be a compact neighborhood of zero in $E$. Suppose that $\alpha$ were transcendental over $P$. Then $\left(\alpha^{n}\right)_{n \geqq 1}$ would be an infinite sequence of distinct scalars, so by [16, Theorem 3], there would exist $n \geqq 1$ such that $W=\alpha V \cap \alpha^{2} V \cap \cdots \cap \alpha^{n} V \subseteq V$. Then $\alpha W \subseteq W$, for if $y \in W$, then $y \in V$, whence $\alpha y \in \alpha V$, and also $y \in \alpha V \cap \cdots \cap \alpha^{n-1} V$, whence $\alpha y \in \alpha^{2} V \cap \cdots \cap \alpha^{n} V$. As $E$ is indiscrete, the neighborhood $W$ of zero contains a nonzero vector $w$. Consequently, $\alpha w, \alpha^{2} w, \alpha^{3} w, \ldots$, would all belong to $W$ by what we have just proved, in contradiction to [16, Lemma 5]. Thus every element of $K$ is algebraic over $P$, so by a theorem of Jacobson [8, p. 183], $K$ is commutative and hence is an absolutely algebraic field.

If $K$ is a ring, we shall denote by $K^{*}$ the set of nonzero elements of $K$.

LEMMA 1. Let $K$ be a division ring, and let $E$ be a $K$-vector space. If $V$ is an additive subgroup of $E$, then $\left\{\lambda_{1} V \cap \cdots \cap \lambda_{n} V: \lambda_{1}, \ldots, \lambda_{n} \in K^{*}\right\}$ is a fundamental system of neighborhoods of zero for a topology on $E$ that makes $E$ a topological vector space over the discrete division ring $K$.

Proof. Addition is continuous in both variables as $\lambda_{1} V \cap \cdots \cap \lambda_{n} V$ is a subgroup. Also if $\alpha \in K^{*}$, then $\alpha\left(\alpha^{-1} \lambda_{1} V \cap \cdots \cap \alpha^{-1} \lambda_{n} V\right)=\lambda_{1} V \cap \cdots \cap \lambda_{n} V$; consequently, $x \rightarrow \alpha x$ is continuous at zero. As $K$ is discrete, $(\lambda, x) \rightarrow \lambda x$ is continuous at $(0,0)$ and for each $a \in E, \lambda \rightarrow \lambda a$ is continuous at zero. Hence $E$ is a topological vector space over $K$.

We shall call the topology defined on $E$ in Lemma 1 the $V$-topology on $E$.

THEOREM 2. Let $E$ be a straight locally compact vector space over an infinite discrete field $K$. Then $E$ is totally disconnected and hence contains a compact open additive subgroup. For any compact open subgroup $V$ of $E$, the topology of $E$ is the $V$-topology.

Proof. Since the connected component $F$ of zero in $E$ is a closed subspace, $F$ is a straight locally compact connected $K$-vector space. If $F$ were not the zero 
subspace, then $F$ would not be compact since it would contain an infinite discrete (and therefore closed) subspace, so by a theorem of Pontrjagin [13, Lemma 2, p. 153], $F$ would contain a nonzero element whose additive order is infinite, in contradiction to Theorem 1. Therefore $F=\{0\}$, so $E$ is totally disconnected. Consequently, the compact open additive subgroups of $E$ form a fundamental system of neighborhoods of zero [3, Corollary 1, p. 58]. The final assertion follows from [16, Theorem 3].

If $u$ is a linear form on a topological vector space over a topological division ring whose topology is given by a proper absolute value and if the kernel of $u$ is closed, then $u$ is continuous [6, Theorem 1, p. 26]. The corresponding assertion for linear forms on straight locally compact vector spaces over infinite discrete fields is a consequence of the following theorem.

THEOREM 3. Let $E$ be a straight locally compact vector space over a discrete infinite field $K$. If $M$ is a closed subspace of $E$ satisfying codim $M \leqq \aleph_{0}$, then $M$ is open.

Proof. As $K$ is countable by Theorem $1, E / M$ is a countable set. As $M$ is closed, $E / M$ is locally compact and hence a Baire space [4, Theorem 1, p. 110]. Therefore the locally compact vector space $E / M$ has an isolated point and hence is discrete. Consequently, $M$ is open.

Corollary 1. If $\operatorname{dim} E \leqq \aleph_{0}$, then $E$ is discrete.

Proof. We need only apply the theorem to the zero subspace.

Corollary 2. If $u$ is a linear form on $E$, then $u$ is continuous if and only if the kernel of $u$ is closed.

Proof. The kernel of $u$ is a subspace of codim $\leqq 1$.

CoROLlaRY 3. If $M$ is a closed subspace of E satisfying codim $M \leqq \aleph_{0}$ and if $N$ is any algebraic supplement of $M$, then $N$ is discrete and $E$ is the topological direct sum of $M$ and $N$.

Proof. Let $p$ be the projection on $N$ along $M$. As $p^{-1}(0)=M$ is open by Theorem 3, $p$ is continuous. Hence $E$ is the topological direct sum of $M$ and $N$ [6, Proposition 12, p. 16]. As $\{0\}=M \cap N$ is open in $N, N$ is discrete.

2. Finite-dimensional subspaces. If $\left\{e_{1}, \ldots, e_{n}\right\}$ is a basis of a finite-dimensional Hausdorff vector space $E$ over a complete division ring $K$ topologized by a proper absolute value (or, more generally, over a complete division ring $K$ of type $V$ ), then $\left(\lambda_{1}, \ldots, \lambda_{n}\right) \rightarrow \sum_{k=1}^{n} \lambda_{k} e_{k}$ is a topological isomorphism from $K^{n}$ onto $E$ [6, Theorem 2, p. 27], [9, Theorem 5]. The corresponding statement for finitedimensional straight locally compact vector spaces over infinite discrete fields is also true (Corollary 1 of Theorem 3). Our primary purpose here is to show that the 
corresponding statement for finite-dimensional subspaces of straight locally compact vector spaces over infinite discrete fields is not true in general.

To establish a criterion for the completion of a topological vector space to be straight and locally compact, we need the following lemma. (We shall denote the closure of a set $G$ by $G^{-}$.)

Lemma 2. Let $G$ be a dense subgroup of a compact group $G^{-}$. If $H$ and $K$ are normal open subgroups of $G$, then $(H \cap K)^{-}=H^{-} \cap K^{-}$.

Proof. The kernel of the homomorphism $x \rightarrow x\left(H^{-} \cap K^{-}\right)$from $G$ into $G^{-} /\left(H^{-} \cap K^{-}\right)$is $G \cap H^{-} \cap K^{-}=H \cap K$ as $H$ and $K$ are open and hence closed subgroups of $G$. Consequently, $G /(H \cap K)$ is isomorphic to a subgroup of $G^{-} /\left(H^{-} \cap K^{-}\right)$, so $\left(G^{-}: H^{-} \cap K^{-}\right) \geqq(G: H \cap K)$. But $h: x(H \cap K) \rightarrow x(H \cap K)^{-}$ is an isomorphism from $G /(H \cap K)$ onto a dense subgroup of $G^{-} /(H \cap K)^{-}$ [3, Proposition 21, p. 27]. As $(H \cap K)^{-}$is a neighborhood in $G^{-}$of the neutral element [3, Proposition 7, p. 43], $G^{-} /(H \cap K)^{-}$is compact and discrete and hence finite. Therefore $h$ is bijective, so $\left(G^{-}:(H \cap K)^{-}\right)=(G: H \cap K) \leqq\left(G^{-}: H^{-} \cap K^{-}\right)$. As $(H \cap K)^{-} \subseteq H^{-} \cap K^{-},\left(G^{-}: H^{-} \cap K^{-}\right) \leqq\left(G^{-}:(H \cap K)^{-}\right)$; thus $\left(G^{-}:(H \cap K)^{-}\right)$ $=\left(G^{-}: H^{-} \cap K^{-}\right)<+\infty$, so $(H \cap K)^{-}=H^{-} \cap K^{-}$.

THEOREM 4. Let $K$ be an infinite discrete field, let $E$ be a vector space over $K$, let $V$ be an infinite additive subgroup of $E$, and let $E$ be topologized by the $V$-topology. The completion $E^{\wedge}$ of $E$ is an indiscrete straight locally compact $K$-vector space and $V^{-}$is a compact neighborhood of zero in $E^{\wedge}$ if and only if the following three conditions hold:

$1^{\circ}$. $\bigcap\left\{\lambda V: \lambda \in K^{*}\right\}=\{0\}$.

$2^{\circ}$. If $\left(\lambda_{k}\right)_{k \geqq 1}$ is any infinite sequence of distinct scalars, then there exists $n \geqq 1$ such that $\lambda_{1} V \cap \cdots \cap \lambda_{n} V \subseteq V$.

$3^{\circ}$. For all $\lambda \in K^{*}, V /(V \cap \lambda V)$ is a finite group.

Proof. Sufficiency. The $V$-topology is Hausdorff by $1^{\circ}$, so $E^{\wedge}$ is Hausdorff. To show that $V$ is precompact, it suffices to show that for any $\lambda_{1}, \ldots, \lambda_{n} \in K^{*}$, $V /\left(V \cap \lambda_{1} V \cap \cdots \cap \lambda_{n} V\right)$ is finite, for then $V$ is the union of finitely many cosets of $V \cap \lambda_{1} V \cap \cdots \cap \lambda_{n} V$ [2, Theorem 3, p. 229]. But the kernel of the homomorphism $x \rightarrow\left(x+V \cap \lambda_{1} V, \ldots, x+V \cap \lambda_{n} V\right)$ from $V$ into $\prod_{i=1}^{n}\left(V /\left(V \cap \lambda_{i} V\right)\right)$, a finite group by $3^{\circ}$, is $V \cap \lambda_{1} V \cap \cdots \cap \lambda_{n} V$. Consequently, $V /\left(V \cap \lambda_{1} V \cap \cdots \cap \lambda_{n} V\right)$ is isomorphic to a subgroup of a finite group and hence is finite. Therefore $V^{-}$is a compact neighborhood of zero in $E^{\wedge}$. Moreover, $E$ is indiscrete, for as $V$ is infinite and $V /\left(V \cap \lambda_{1} V \cap \cdots \cap \lambda_{n} V\right)$ finite, $V \cap \lambda_{1} V \cap \cdots \cap \lambda_{n} V \neq\{0\}$. Consequently $E^{\wedge}$ is indiscrete.

Next we shall show that for any infinite sequence $\left(\beta_{k}\right)_{k \geqq 1}$ of nonzero scalars, $\left(\beta_{1} V \cap \cdots \cap \beta_{n} V\right)_{n \geqq 1}$ is a fundamental system of neighborhoods of zero in $E$. Indeed, for any $\alpha \in K^{*}$ there exists $m \geqq 1$ such that $\alpha^{-1} \beta_{1} V \cap \cdots \cap \alpha^{-1} \beta_{m} V \subseteq V$ 
by $2^{\circ}$, whence $\beta_{1} V \cap \cdots \cap \beta_{m} V \subseteq \alpha V$. Consequently for any $\alpha_{1}, \ldots, \alpha_{s} \in K^{*}$, there exists $n \geqq 1$ such that $\beta_{1} V \cap \cdots \cap \beta_{n} V \subseteq \alpha_{1} V \cap \cdots \cap \alpha_{s} V$.

To show that $E^{\wedge}$ is straight, let $z \in E^{\wedge}$, and assume that there exist infinitely many nonzero scalars, $\lambda_{1}, \lambda_{2}, \ldots$, such that $\lambda_{k} z \in V^{-}$for all $k \geqq 1$. Then by Lemma 2 , applied to $G^{-}=\left(\lambda_{1}^{-1} V+\cdots+\lambda_{n}^{-1} V\right)^{-}$,

$$
\begin{aligned}
z \in \bigcap_{n=1}^{\infty}\left(\lambda_{1}^{-1} V^{-} \cap \cdots \cap \lambda_{n}^{-1} V^{-}\right) & =\bigcap_{n=1}^{\infty}\left(\left(\lambda_{1}^{-1} V\right)^{-} \cap \cdots \cap\left(\lambda_{n}^{-1} V\right)^{-}\right) \\
& =\bigcap_{n=1}^{\infty}\left(\lambda_{1}^{-1} V \cap \cdots \cap \lambda_{n}^{-1} V\right)^{-}=\{0\}
\end{aligned}
$$

as $E^{\wedge}$ is Hausdorff and as $\left(\left(\lambda_{1}^{-1} V \cap \cdots \cap \lambda_{n}^{-1} V\right)^{-}\right)_{n \geqq 1}$ is a fundamental system of neighborhoods of zero in $E^{\wedge}$ by what we have just proved and [3, Proposition 7, p. 43]. Consequently, if $z$ is a nonzero vector of $E^{\wedge}$, then $K . z \cap V^{-}$is finite; hence $E^{\wedge}$ is straight.

Necessity. Condition $1^{\circ}$ holds as $E^{\wedge}$ is Hausdorff. By [16, Theorem 3], if $\left(\lambda_{k}\right)_{k \geqq 1}$ is any infinite sequence of distinct scalars, there exists $n \geqq 1$ such that $\lambda_{1} V^{-} \cap \ldots$ $\cap \lambda_{n} V^{-} \subseteq V^{-}$, whence $\left(\lambda_{1} V^{-} \cap \cdots \cap \lambda_{n} V^{-}\right) \cap E \subseteq V^{-} \cap E=V$. But by Lemma 2, applied to $G^{-}=\left(\lambda_{1} V+\cdots+\lambda_{n} V\right)^{-}, \quad \bigcap_{i=1}^{n} \lambda_{i} V^{-}=\bigcap_{i=1}^{n}\left(\lambda_{i} V\right)^{-}=\left(\bigcap_{i=1}^{n} \lambda_{i} V\right)^{-}$, whence $\left(\bigcap_{i=1}^{n} \lambda_{i} V^{-}\right) \cap E=\left(\bigcap_{i=1}^{n} \lambda_{i} V\right)^{-} \cap E=\bigcap_{i=1}^{n} \lambda_{i} V$. Thus $2^{\circ}$ holds. To show that $3^{\circ}$ holds, it suffices to note that $V /(V \cap \lambda V)$ is isomorphic to a subgroup of $V^{-} /(V \cap \lambda V)^{-}$[3, Proposition 21, p. 27] and that $V^{-} /(V \cap \lambda V)^{-}$is compact and discrete and hence finite since $(V \cap \lambda V)^{-}$is a neighborhood of zero in $E^{\wedge}$.

Let $K$ be an infinite absolutely algebraic field of prime characteristic $p$, equipped with the discrete topology. To show that there exists a straight locally compact $K$-vector space having a dense two-dimensional subspace, we need only find a subgroup $V$ of a two-dimensional $K$-vector space $E$ satisfying the conditions of Theorem 4. To do so we shall first construct an automorphism $\sigma$ of the additive group $K$.

As $K$ is absolutely algebraic of prime characteristic, $K$ is the union of a strictly increasing sequence $\left(K_{n}\right)_{n \geqq 0}$ of finite fields. We may assume that $K_{0}$ has more than two elements. For each $n \geqq 1$, there exists $\alpha_{n} \in K_{n}$ such that $K_{n}=K_{n-1}\left(\alpha_{n}\right)$ by the Theorem of the Primitive Element [15, Theorem 49.9, p. 546].

We define recursively a sequence of mappings $\left(\sigma_{n}\right)_{n \geqq 0}$ such that each $\sigma_{n}$ is an automorphism of the additive group $K_{n}$ and, if $n \geqq 1, \sigma_{n}$ is an extension of $\sigma_{n-1}$. Let $\sigma_{0}$ be the identity automorphism of $K_{0}$. Assume that $\sigma_{n-1}$ is defined. As $\beta^{-1} \sigma_{n-1}(\beta)=\beta^{-1} \sigma_{0}(\beta)=1$ for all $\beta \in K_{0}^{*}$, a set having more than one element, the function $\beta \rightarrow \beta^{-1} \sigma_{n-1}(\beta)$ on $K_{n-1}^{*}$ is not injective and hence not surjective, so there exists $\gamma_{n-1} \in K_{n-1}^{*}$ such that $\gamma_{n-1} \neq \beta^{-1} \sigma_{n-1}(\beta)$ for all $\beta \in K_{n-1}^{*}$. Let $q=\left[K_{n}: K_{n-1}\right]$. Then $1, \alpha_{n}, \ldots, \alpha_{n}^{q-1}$ is a basis of the $K_{n-1}$-vector space $K_{n}$. We define $\sigma_{n}$ on $K_{n}$ by

$$
\sigma_{n}\left(\sum_{k=0}^{q-1} \lambda_{k} \alpha_{n}^{k}\right)=\sigma_{n-1}\left(\lambda_{0}\right)+\gamma_{n-1}\left(\sum_{k=1}^{q-1} \lambda_{k} \alpha_{n}^{k}\right)
$$


for all $\lambda_{0}, \ldots, \lambda_{q-1} \in K_{n-1}$. Clearly $\sigma_{n}$ is an automorphism of the additive group $K_{n}$ that extends $\sigma_{n-1}$. Consequently we may define $\sigma$ on $K$ by $\sigma(\zeta)=\sigma_{n}(\zeta)$ where $n$ is such that $\zeta \in K_{n}$. Then $\sigma$ is clearly an automorphism of the additive group $K$. Also, we define $L_{n}$ to be the additive subgroup $K_{n-1} \alpha_{n}+\cdots+K_{n-1} \alpha_{n}^{q-1}$ of $K_{n}$. Thus the additive group $K_{n}$ is the direct sum of $K_{n-1}$ and $L_{n}$, and for each $n \geqq 1$, the additive group $K$ is the direct sum of $K_{n-1}$ and $\sum_{m \geqq n} L_{m}$.

LeMmA 3. Let $n \geqq 1$, and let $\zeta, \beta \in K_{n}$. If $\zeta \notin K_{n-1}$ and if $\beta \notin L_{n}$, then $\left.\sigma(\beta \zeta) \neq \sigma^{\prime} \beta\right) \zeta$.

Proof. Let $q=\left[K_{n}: K_{n-1}\right]$. For each $k \in[1, q-1]$ let

$$
\alpha_{n}^{q-1+k}=\lambda_{k, 0}+\lambda_{k, 1} \alpha_{n}+\cdots+\lambda_{k, q-1} \alpha_{n}^{q-1}
$$

where $\lambda_{k, 0}, \ldots, \lambda_{k, q-1} \in K_{n-1}$. Similarly, let

$$
\begin{aligned}
& \zeta=\zeta_{0}+\zeta_{1} \alpha_{n}+\cdots+\zeta_{q-1} \alpha_{n}^{q-1}, \\
& \beta=\beta_{0}+\beta_{1} \alpha_{n}+\cdots+\beta_{q-1} \alpha_{n}^{q-1},
\end{aligned}
$$

where $\zeta_{0}, \ldots, \zeta_{q-1}, \beta_{0}, \ldots, \beta_{q-1} \in K_{n-1}$. By hypothesis, $\beta_{0} \neq 0$ and $\zeta_{m} \neq 0$ for some $m \geqq 1$. The coefficient of $\alpha_{n}^{m}$ in $\beta \zeta$ is

$$
\beta_{0} \zeta_{m}+\beta_{1} \zeta_{m-1}+\cdots+\beta_{m} \zeta_{0}+\sum_{k=1}^{q-1}\left(\beta_{k} \zeta_{q-1}+\beta_{k+1} \zeta_{q-2}+\cdots+\beta_{q-1} \zeta_{k}\right) \lambda_{k, m},
$$

so the coefficient of $\alpha_{n}^{m}$ in $\sigma(\beta \zeta)$ is $\gamma_{n-1}$ times that scalar. Also, the coefficient of $\alpha_{n}^{m}$ in $\sigma(\beta) \zeta$ is

$$
\sigma\left(\beta_{0}\right) \zeta_{m}+\gamma_{n-1} \beta_{1} \zeta_{m-1}+\cdots+\gamma_{n-1} \beta_{m} \zeta_{0}+\sum_{k=1}^{q-1} \gamma_{n-1}\left(\beta_{k} \zeta_{q-1}+\cdots+\beta_{q-1} \zeta_{k}\right) \lambda_{k, m} .
$$

Therefore the coefficient of $\alpha_{n}^{m}$ in $\sigma(\beta \zeta)-\sigma(\beta) \zeta$ is $\gamma_{n-1} \beta_{0} \zeta_{m}-\sigma\left(\beta_{0}\right) \zeta_{m}$, which is not zero since $\zeta_{m} \neq 0$ and since $\gamma_{n-1} \beta_{0} \neq \sigma\left(\beta_{0}\right)$ as $\beta_{0} \neq 0$. Therefore $\sigma(\beta \zeta) \neq \sigma(\beta) \zeta$.

LEMMA 4. If $\zeta \in K_{n}$ and if $\beta \in \sum_{m \geqq n+1} L_{m}$, then $\sigma(\beta \zeta)=\sigma(\beta) \zeta$.

Proof. By the additivity of $\sigma$, it suffices to prove that for each $m \geqq n+1$, $\sigma(\beta \zeta)=\sigma(\beta) \zeta$ for all $\beta \in L_{m}$. Let $\beta=\sum_{k=1}^{q-1} \beta_{k} \alpha_{m}^{k} \in L_{m}$, where $q=\left[K_{m}: K_{m-1}\right]$ and $\beta_{1}, \ldots, \beta_{q-1} \in K_{m-1}$. Then $\zeta \in K_{m-1}$, so

$$
\begin{aligned}
\sigma(\beta \zeta) & =\sigma\left(\sum_{k=1}^{q-1} \beta_{k} \zeta \alpha_{m}^{k}\right)=\gamma_{m-1}\left(\sum_{k=1}^{q-1} \beta_{k} \zeta \alpha_{m}^{k}\right) \\
& =\zeta \gamma_{m-1}\left(\sum_{k=1}^{q-1} \beta_{k} \alpha_{m}^{k}\right)=\zeta \sigma(\beta) .
\end{aligned}
$$

Let $\left\{e_{1}, e_{2}\right\}$ be a basis of a two-dimensional $K$-vector space $E$, and let

$$
V=\left\{\beta e_{1}+\sigma(\beta) e_{2}: \beta \in K\right\} .
$$

Clearly $V$ is an infinite additive subgroup of $E$. Also, for each $n \geqq 1$ let

$$
V_{n}=\left\{\beta e_{1}+\sigma(\beta) e_{2}: \beta \in \sum_{m \geqq n} L_{m}\right\} \text {. }
$$


Lemma 5. Let $n \geqq 1$, and let $\zeta \in K_{n}$. If $\zeta \notin K_{n-1}$, then

$$
V_{n+1} \subseteq V \cap \zeta^{-1} V \subseteq V_{n}
$$

Proof. To show that $V_{n+1} \subseteq V \cap \zeta^{-1} V$, let $\beta \in \sum_{m \geqq n+1} L_{m}$. By Lemma 4, $\sigma(\beta \zeta)=\sigma(\beta) \zeta$, so $\beta e_{1}+\sigma(\beta) e_{2}=\zeta^{-1}\left[\beta \zeta e_{1}+\sigma(\beta \zeta) e_{2}\right] \in \zeta^{-1} V$. To show that $V \cap \zeta^{-1} V$ $\subseteq V_{n}$, let $\beta e_{1}+\sigma(\beta) e_{2} \in \zeta^{-1} V$. Then $\zeta \beta e_{1}+\zeta \sigma(\beta) e_{2}=\gamma e_{1}+\sigma(\gamma) e_{2}$ for some $\gamma \in K$, whence $\zeta \beta=\gamma$ and $\zeta \sigma(\beta)=\sigma(\gamma)$. Therefore $\sigma(\beta \zeta)=\sigma(\beta) \zeta$. Let $\beta=\beta_{1}+\beta_{2}$ where $\beta_{1} \in K_{n}, \beta_{2} \in \sum_{m \geqq n+1} L_{m}$. By Lemma $4, \sigma\left(\beta_{2} \zeta\right)=\sigma\left(\beta_{2}\right) \zeta$, so by the additivity of $\sigma$, $\sigma\left(\beta_{1} \zeta\right)=\sigma\left(\beta_{1}\right) \zeta$. By Lemma 3, $\beta_{1} \in L_{n}$. Therefore $\beta \in \sum_{m \geqq n} L_{m}$, so $\beta e_{1}+\sigma(\beta) e_{2} \in V_{n}$.

We may now show that $V$ satisfies the three conditions of Theorem 4. By Lemma $5,1^{\circ}$ holds. We observe also that if $\zeta \in K_{0}^{*}$, then $V \subseteq \zeta^{-1} V$. Indeed, for any $\beta \in K$, $\beta e_{1}+\sigma(\beta) e_{2}=\zeta^{-1}\left[\zeta \beta+\zeta \sigma(\beta) e_{2}\right]$. If $\beta \in \sum_{n \geqq 1} L_{n}$, then $\zeta \sigma(\beta)=\sigma(\zeta \beta)$ by Lemma 4, and hence $\beta e_{1}+\sigma(\beta) e_{2} \in \zeta^{-1} V$; if $\beta \in K_{0}$, then $\sigma(\beta)=\beta$ and $\sigma(\zeta \beta)=\zeta \beta$, whence $\zeta \sigma(\beta)=\sigma(\zeta \beta)$ and thus $\beta e_{1}+\sigma(\beta) e_{2} \in \zeta^{-1} V$; therefore by additivity, $V \subseteq \zeta^{-1} V$. To prove $2^{\circ}$, let $\left(\lambda_{k}\right)_{k \geqq 1}$ be an infinite sequence of distinct nonzero scalars, and let $\zeta=\lambda_{1}, \zeta_{k}=\lambda_{1} \lambda_{k+1}^{-1}$ for all $k \geqq 1$. We shall prove that $V \cap \zeta_{1}^{-1} V \cap \cdots \cap \zeta_{m}^{-1} V$ $\subseteq V \cap \zeta^{-1} V$ for some $m \geqq 1$. By the preceding, we may assume that $\zeta \notin K_{0}$. Let $n \geqq 1$ be such that $\zeta \in K_{n}-K_{n-1}$. As $K_{n+1}$ is finite, there exists $m \geqq 1$ such that $\zeta_{m} \in K_{s}-K_{s-1}$ for some $s \geqq n+1$. Consequently,

$$
V \cap \zeta_{1}^{-1} V \cap \cdots \cap \zeta_{m}^{-1} V \subseteq V \cap \zeta_{m}^{-1} V \subseteq V_{s} \subseteq V_{n+1} \subseteq V \cap \zeta^{-1} V
$$

by Lemma 5. Thus $V \cap \lambda_{1}^{-1} \lambda_{2} V \cap \cdots \cap \lambda_{1}^{-1} \lambda_{m+1} V \subseteq V \cap \lambda_{1}^{-1} V$, whence

$$
\lambda_{1} V \cap \lambda_{2} V \cap \cdots \cap \lambda_{m+1} V \subseteq \lambda_{1} V \cap V .
$$

Therefore $2^{\circ}$ holds. To prove $3^{\circ}$, let $\lambda \in K^{*}$. By the preceding, we may assume that $\lambda \notin K_{0}$. Let $n \geqq 1$ be such that $\lambda \in K_{n}-K_{n-1}$. As $\beta \rightarrow \beta e_{1}+\sigma(\beta) e_{2}$ is an isomorphism from $K$ onto $V$, by Lemma 5 we obtain $(V: V \cap \lambda V) \leqq\left(V: V_{n+1}\right)=\left(K: \sum_{m \geqq n+1} L_{m}\right)$ $=$ cardinality of $K_{n}<\infty$. Thus we have proved:

THEOREM 5. If $K$ is an infinite absolutely algebraic field of prime characteristic, equipped with the discrete topology, then there is an indiscrete straight locally compact $K$-vector space that contains a dense two-dimensional subspace.

A general question of interest is the following: If $K$ is a topological division ring, is every straight Hausdorff two-dimensional vector space all of whose onedimensional subspaces are closed isomorphic to $K^{2}$ ? By [17, Theorem 6] the answer is yes if every Hausdorff $K$-vector space is straight, and in particular if $K$ is of type $V$ [17, Theorem 2]; we have just seen that it is no if $K$ is an infinite discrete absolutely algebraic field of prime characteristic.

If $E$ is a topological vector space, the topological dual $E^{*}$ of $E$ is the space of all continuous linear forms on $E$; $E^{*}$ is total if for every nonzero $x \in E$ there exists $u \in E^{*}$ such that $u(x) \neq 0$.

THEOREM 6. If $E$ is an indiscrete straight locally compact vector space over an 
infinite discrete field $K$ and if $E$ contains a dense subspace $M$ every proper subspace of which is discrete, then $E^{*}=\{0\}$.

Proof. It suffices to show that $M^{*}=\{0\}$. Suppose that there existed a nonzero $u \in M^{*}$; then the kernel of $u$ would be a proper open subspace of $M$ and hence would be discrete and open; thus $M$ itself would be discrete and hence closed, so $E$ would be $M$ and hence would be discrete, a contradiction.

Thus, for example, there are no nonzero continuous linear forms on the spaces mentioned in Theorem 5.

THEOREM 7. If $E$ is a straight locally compact vector space over an infinite discrete field $K$ and if $E^{*}$ is total, then every finite-dimensional subspace of $E$ is discrete.

Proof. If not, there would exist an indiscrete finite-dimensional subspace $M$ each of whose proper subspaces was discrete; consequently $M^{-*}=\{0\}$ by Theorem 6, so $E^{*}$ would not be total, a contradiction.

It is an open question whether the converse of Theorem 7 holds, that is, whether the topological dual of a straight locally compact $K$-vector space all of whose finite-dimensional subspaces are discrete is total. The space $E$ constructed in [16, Example 1] has a total topological dual, for the projection functions $e_{m}$ : $\left(\lambda_{n}\right) \rightarrow \lambda_{m}$ are clearly continuous; thus every finite-dimensional subspace of $E$ is discrete.

3. Compactly generated locally compact vector spaces. For what pairs of straight locally compact vector spaces over infinite discrete fields are the Open Homomorphism Theorem and the Closed Graph Theorem valid? The answer concerns compactly generated vector spaces.

Definition. A topological vector space is compactly generated if it contains a set of generators that is compact.

Thus every Hausdorff finite-dimensional vector space is compactly generated, and a discrete vector space is compactly generated if and only if it is finitedimensional.

THEOREM 8. Let $K$ be a topological division ring of prime characteristic. If $S$ is a compact subset of a locally compact, totally disconnected $K$-vector space $E$, then there is a compact open subgroup $V$ of $E$ that contains $S$. In particular, if $E$ is a compactly generated, locally compact, totally disconnected $K$-vector space, then $E$ contains a compact open subgroup $V$ that generates $E$.

Proof. Let $P$ be the prime subfield of $K$. By [3, Corollary 1, p. 58], $E$ contains a compact open subgroup $W$. As $S$ is compact, there exist $a_{1}, \ldots, a_{n} \in E$ such that $S \subseteq \bigcup_{i=1}^{n}\left(a_{i}+W\right)$. Then $V=P . a_{1}+\cdots+P . a_{n}+W$ is a compact open subgroup of $E$ that contains $S$.

Theorem 8 applies, of course, to straight locally compact vector spaces over infinite discrete fields by Theorem 2 . 
THEOREM 9. Let $E$ and $F$ be straight locally compact vector spaces over an infinite discrete field $K$, and let $u$ be a linear transformation from $E$ into $F$. If $F$ is compactly generated and if the graph of $u$ is closed, then $u$ is continuous. If $E$ is compactly generated and if $u$ is a continuous epimorphism, then $u$ is open.

Proof. By Theorem 2, $E$ and $F$ are complete and metrizable. If $F$ is compactly generated, by Theorem $8 F$ contains a compact open subgroup $V$ that generates $E$; thus

$$
F=\bigcup\left\{\lambda_{1} V+\cdots+\lambda_{n} V: \lambda_{1}, \ldots, \lambda_{n} \in K\right\},
$$

a countable union of compact sets since $K$ is countable by Theorem 1 ; consequently, $F$ is $\sigma$-compact and hence is a Lindelöf space. Therefore the two assertions follow from versions of the Closed Graph and Open Homomorphism Theorems for topological groups [11, p. 213].

The hypothesis in Theorem 9 that the spaces be compactly generated cannot be omitted, even if we assume further that the spaces are indiscrete. Indeed, let $E$ be an indiscrete straight locally compact $K$-vector space, and let $E_{0}$ be $E$ equipped with the discrete topology. Then the graph of the identity mapping $u$ from $E \times E$ into $E \times E_{0}$ is closed, but $u$ is not continuous; also $u^{-1}$ is continuous but not open.

THEOREM 10. A straight locally compact vector space $E$ over an infinite discrete field $K$ is the topological direct sum of a compactly generated locally compact subspace and a discrete subspace. Indeed, if $F$ is the subspace generated by a compact neighborhood of zero and if $M$ is any algebraic supplement of $F$, then $F$ is locally compact, $M$ is discrete, and $E$ is the topological direct sum of $F$ and $M$.

Proof. Let $p$ be the projection on $M$ along $F$. Then $p^{-1}(0) \supseteq F$, a neighborhood of zero, so $p$ is continuous. Consequently, $E$ is the topological direct sum of $F$ and $M$ [6, Proposition 12, p. 16]. Also as $F \cap M=\{0\}, M$ is discrete. Clearly $F$ is locally compact.

THEOREM 11. Let $E$ be a compactly generated straight locally compact vector space over an infinite discrete field $K$, and let $M$ and $N$ be supplementary subspaces of $E$. Then $E$ is the topological direct sum of $M$ and $N$ if and only if $M$ and $N$ are closed and compactly generated.

Proof. Necessity. The projection $p$ on $M$ along $N$ is continuous. Consequently, if $E$ is generated by the compact set $V$, then $M$ is generated by the compact set $p(V)$. Similarly, $N$ is compactly generated. Sufficiency. Let $A$ be the algebraic isomorphism $(x, y) \rightarrow x+y$ from $M \times N$ onto $E$. As $M$ and $N$ are compactly generated straight locally compact spaces, so is $M \times N$. Consequently as $A$ is continuous, $A$ is open and hence a topological isomorphism by Theorem 9 . Thus $E$ is the topological direct sum of $M$ and $N$.

THEOREM 12. Let $V$ and $W$ be compact open subgroups of a straight locally compact vector space $E$ over an infinite discrete field $K$, and let $E_{V}$ and $E_{W}$ be the 
subspaces of $E$ generated by $V$ and $W$ respectively. If $V \subseteq W$, then $E_{W} / E_{V}$ is finitedimensional.

Proof. As $W$ is compact and $V$ open, $W / V$ is a compact discrete group and hence is finite. Thus there exist $a_{1}, \ldots, a_{m} \in W$ such that $W=\bigcup_{i=1}^{m}\left(a_{i}+V\right)$. Let $M$ be the subspace generated by $a_{1}, \ldots, a_{m}$. It suffices to show that $E_{W}=M+E_{V}$. Let $z \in E_{W}$. Then there exist $\lambda_{1}, \ldots, \lambda_{n} \in K$ such that $z \in \lambda_{1} W+\cdots+\lambda_{n} W$, so $z=\lambda_{1} w_{1}+\cdots+\lambda_{n} w_{n}$ where $\lambda_{i} \in K, w_{i} \in W$ for all $i \in[1, n]$. For each $i \in[1, n]$ let $w_{i}=a_{q(i)}+v_{i}$ where $q(i) \in[1, m]$ and $v_{i} \in V$; then

$$
z=\sum_{i=1}^{n} \lambda_{i} a_{q(i)}+\sum_{i=1}^{n} \lambda_{i} v_{i} \in M+E_{V}
$$

THEOREM 13. If $E$ is a straight locally compact vector space over an infinite discrete field $K$, then $E$ is compactly generated if and only if $E^{*}$ is countable.

Proof. Necessity. By Theorem $8, E$ is generated by a compact open subgroup, and by Theorems 1 and 2 there is a sequence $\left(V_{n}\right)_{n \geqq 1}$ of compact open subgroups that is a fundamental system of neighborhoods of zero. For each $n \geqq 1$ let $E_{n}$ be the subspace generated by $V_{n}$. By Theorem $12, E / E_{n}$ is finite-dimensional. Consequently, as $K$ is countable, the space of all linear forms whose kernel contains $E_{n}$ is countable as it is isomorphic to the dual of $E / E_{n}$. But the kernel of a continuous linear form on $E$ is an open subspace and hence contains $E_{n}$ for some $n \geqq 1$. Thus $E^{*}$ is countable.

Sufficiency. By Theorem 10,E is the topological direct sum of a compactly generated subspace $F$ and a discrete subspace $M$; let $p$ be the projection on $M$ along $F$. Then $u \rightarrow u p$ is a monomorphism from the space $M^{*}$ of all linear forms on $M$ into $E^{*}$. If $M$ were infinite-dimensional, then $M^{*}$ and hence also $E^{*}$ would be uncountable, a contradiction. Therefore $M$ is finite-dimensional. Consequently if $C$ is a compact set of generators for $F$ and if $B$ is a basis of $M$, then $C \cup B$ is a compact set of generators for $E$.

4. Locally compact algebras of linear operators. For any topological vector space $E$ it is natural to inquire whether the algebra of all continuous linear operators on $E$ is dense. One answer to this question involves straightness and the totality of the topological dual.

THEOREM 14. Let $E$ be a nonzero topological vector space over a topological field $K$, and let $A$ be the algebra of all continuous linear operators on $E$. The following statements are equivalent:

$1^{\circ}$. $E^{*}$ is total.

$2^{\circ}$. E is straight, and $A$ is a dense algebra of linear operators that contains nonzero linear operators of finite rank.

$3^{\circ} . E$ is straight, and every finite-dimensional subspace of $E$ has a topological supplement. 
Proof. For each $a \in E$ let $f_{a}: \lambda \rightarrow \lambda a, \lambda \in K$. To show that $1^{\circ}$ implies $2^{\circ}$ let $a$ be a nonzero vector of $E$. By $1^{\circ}$ there exists $u \in E^{*}$ such that $u(a)=1$. Then the restriction of $u$ to $K$. $a$ is $f_{a}^{-1}$, so $f_{a}$ is a homeomorphism from $K$ onto $K . a$; therefore $E$ is straight. Moreover, for any $b \in E, v_{a, b}=f_{b} \circ u \in A$, the range of $v_{a, b}$ is $K . b$, and $v_{a, b}(a)=b$. Thus $A$ is a primitive algebra of endomorphisms of the abelian group $E$. Let $g$ belong to the centralizer of $A$ in the ring of all endomorphisms of the abelian group $E$. As $g \circ v_{a, a}=v_{a, a} \circ g$ for all nonzero $a \in E$, for each $a \in E$ there exists $\lambda_{a} \in K$ such that $g(a)=\lambda_{a} a$. If $b$ is a scalar multiple of $a$, clearly $\lambda_{b}=\lambda_{a}$; if $\{a, b\}$ is linearly independent, then $\lambda_{a} a+\lambda_{b} b=g(a)+g(b)=g(a+b)=\lambda_{a+b} a$ $+\lambda_{a+b} b$, so $\lambda_{a}=\lambda_{a+b}=\lambda_{b}$. Therefore $g$ is a scalar multiple of the identity linear operator. Consequently by the Chevalley-Jacobson Density Theorem [14, p. 344], $A$ is a dense algebra of linear operators, and we have already seen that $A$ contains nonzero linear operators of finite rank.

By a well-known theorem of algebra [14, Exercise 32.27, p. 354], $2^{\circ}$ implies that if $M$ is a finite-dimensional subspace of $E$, there is a continuous projection from $E$ onto $M$, whence $M$ admits a topological supplement [6, Proposition 12, p. 16].

To show that $3^{\circ}$ implies $1^{\circ}$, let $a$ be a nonzero vector of $E$. By $3^{\circ}, K . a$ has a topological supplement, and hence by the theorem just cited there is a continuous projection $p$ from $E$ onto $K . a$. Then $u=f_{a}^{-1} \circ p$ is continuous as $E$ is straight, so $u \in E^{*}$ and $u(a)=1$.

COROLlARY. Let $E$ and $F$ be topological vector spaces over a topological field $K$, and let $M$ be a finite-dimensional subspace of $E$. If $E^{*}$ is total and if $u$ is a continuous linear transformation from $M$ into $F$, then there is a continuous linear transformation $v$ from $E$ into $F$ that extends $u$.

Proof. By $3^{\circ}$ of Theorem 14 there is a continuous projection $p$ from $E$ onto $M$. Consequently, $v=u \circ p$ is the desired extension of $u$.

THEOREM 15. Let $E$ be a straight locally compact vector space over an infinite discrete field $K$, and let $A$ be the algebra of all continuous linear operators on $E$, equipped with the compact-open topology.

$1^{\circ}$. A is a complete, straight topological K-algebra.

$2^{\circ} . A$ is locally compact if and only if $E$ is compactly generated.

$3^{\circ}$. If $E$ is compactly generated, then $A$ is separable and metrizable.

$4^{\circ}$. If $E$ is indiscrete and if $E^{*}$ is total, then $A$ is indiscrete.

Proof. As $E$ is complete and as $A$ is closed in the space of all continuous functions from $E$ into $E$, equipped with the compact-open topology, $A$ is a complete topological algebra by well-known theorems [5, Proposition 9, p. 47, Corollary 3, p. 20]. To show that $A$ is straight, let $u$ be a nonzero linear operator in $A$, and let $x \in E$ be such that $u(x) \neq 0$. If $V$ is a compact neighborhood of zero in $E$, then $K . u$ $\cap\{v \in A: v(x) \in V\}=\{\lambda u: \lambda u(x) \in V\}$ is finite by [16, Lemma 5], so $K . u$ is discrete as $\{v \in A: v(x) \in V\}$ is a neighborhood of zero in $A$. 
Assume next that $A$ is locally compact. By Theorem 8 , every compact subset of $E$ is contained in a compact open subgroup; hence there exist compact open subgroups $U$ and $V$ of $E$ such that $W=\{u \in A: u(U) \subseteq V\}$ is compact. Let $F$ be the subspace of $E$ generated by $U$, and let $M$ be an algebraic supplement of $F$. Then $E$ is the topological direct sum of $F$ and $M$ by Theorem 10, and consequently the projection $p$ on $M$ along $F$ is continuous. Clearly $\lambda p \in W$ for all $\lambda \in K$, so $p=0$ by $1^{\circ}$ and [16, Lemma 5], and hence $M=\{0\}$. Thus $F=E$, so $E$ is compactly generated.

Next, suppose that $E$ is compactly generated, and let $V$ be a compact open subgroup that generates $E$. Then $E=\bigcup_{n \geqq 1}\left(\lambda_{1} V+\cdots+\lambda_{n} V\right)$ where $\left(\lambda_{k}\right)_{k \geqq 1}$ is an enumeration of the elements of $K$, so $E$ is locally compact, metrizable and $\sigma$-compact; hence $E$ is also separable [4, Corollary, p. 43], and consequently $A$ is separable and metrizable [5, Corollary, p. 34, and Corollary, p. 41]. Let $W=\{u \in A: u(V) \subseteq V\}$. Then $W$ is a closed neighborhood of zero in $A$. Also $W$ is equicontinuous since $W\left(\lambda_{1} V \cap \cdots \cap \lambda_{n} V\right) \subseteq \lambda_{1} V \cap \cdots \cap \lambda_{n} V$ for all $\lambda_{1}, \ldots, \lambda_{n} \in K^{*}$. To show that $W$ is compact, therefore, it suffices by Ascoli's Theorem [5, Corollary 3, p. 32] to show that $W(x)$ is relatively compact for each $x \in E$. But if $x \in E$, then there exist $\lambda_{1}, \ldots, \lambda_{m} \in K^{*}$ such that $x \in \lambda_{1} V+\cdots+\lambda_{m} V$, whence $W(x) \subseteq \lambda_{1} V+\cdots+\lambda_{m} V$, a compact set. Therefore $A$ is locally compact.

To prove $4^{\circ}$, let $U$ be a compact subset of $E, V$ a neighborhood of zero in $E$, and let $W=\{u \in A: u(U) \subseteq V\}$. By Theorem 14, $A$ is dense and contains a projection $p$ on a one-dimensional subspace K.c. As $K . c$ is discrete, $p(U)$ is finite; let $p(U)=\left\{\beta_{1} c, \ldots, \beta_{n} c\right\}$. The function $x \rightarrow\left(\beta_{1} x, \ldots, \beta_{n} x\right)$ from $E$ into $E^{n}$ is continuous, and hence as $E$ is indiscrete, there exists a nonzero vector $b \in E$ such that $\beta_{1} b, \ldots, \beta_{n} b \in V$. Also as $A$ is dense there exists $u \in A$ such that $u(c)=b$. Consequently, $u \circ p$ is a nonzero member of $W$.

We recall briefly the basic facts concerning primitive rings having minimal left ideals. If $A$ is such a ring, then $A$ contains a minimal idempotent $e, e A e$ is a division ring, the additive group $A e$ becomes a right $e A e$-vector space by defining scalar multiplication to be the restriction of multiplication on $A$ to $A e \times e A e$, and similarly the additive group $e A$ becomes a left $e A e$-vector space by defining scalar multiplication to be the restriction of multiplication on $A$ to $e A e \times e A$. For each $a \in A, a_{L}: x \rightarrow a x$ is a linear operator on the right vector space $A e$, and $a_{R}: x \rightarrow x a$ is a linear operator on the left vector space $e A$; finally, $a \rightarrow a_{L}$ is an isomorphism from $A$ onto a dense ring $A_{L}$ of linear operators on $A e$, and $a \rightarrow a_{R}$ is an antiisomorphism from $A$ onto a dense ring $A_{R}$ of linear operators on $e A$, and both $A_{L}$ and $A_{R}$ contain nonzero linear operators of finite rank [8, Chapters 2 and 4]. If in addition $A$ is a locally compact ring, then the induced topology on $e A e$ makes $e A e$ a locally compact division ring [12, Theorem 3], [10, Theorems 7-9], and the induced topologies on $A e$ and $e A$ make them straight locally compact vector spaces over $e A e$ [16, Lemma 1]. If $A_{L}$ and $A_{R}$ are topologized so that $a \rightarrow a_{L}$ and $a \rightarrow a_{R}$ respectively are homeomorphisms, then $A_{L}$ and $A_{R}$ are locally compact 
rings whose topologies are stronger than the compact-open topology, since $(u, x) \rightarrow u(x)$ is continuous from $A_{L} \times A e$ into $A e$ and also from $A_{R} \times e A$ into $e A$ [5, Corollary 1, p. 46].

Before giving examples of compactly generated locally compact primitive algebras having minimal left ideals, we recall that by a theorem of Kaplansky [9, Theorem 8], the topology of an indiscrete locally compact division ring $D$ is defined by an absolute value, its center $C$ is indiscrete, and $[D: C]<+\infty$. Consequently, if $K$ is an infinite subfield of $D$, then $K$ is indiscrete; for if there exists $z \in K^{*}$ such that $|z| \neq 1$, then either $z^{n} \rightarrow 0$ or $z^{-n} \rightarrow 0$, and thus $K$ is indiscrete; hence if $K$ were discrete, $K$ would be a closed subset of the closed unit ball, a compact set, which is impossible.

Let $D$ be an indiscrete locally compact division algebra over an infinite topological field $K$, and let $A$ be the ring of all linear operators on a finite-dimensional topological $D$-vector space $E$. As $D$ is finite-dimensional over its center $C, A$ is a finite-dimensional $C$-algebra, and hence as $C$ is indiscrete, $A$ has a unique Hausdorff topology making it a topological $C$-algebra, namely, the compact-open topology. As $K$ is algebraically isomorphic to a subfield $K^{\prime}$ of $C, A$ is in a natural way a locally compact $K$-algebra. Moreover, $A$ is a compactly generated $K$-algebra, for as $K^{\prime}$ is indiscrete, $K^{\prime}$ contains scalars of arbitrarily large absolute value, so the closed unit ball $B$ of $C$ is a compact set of generators of the $K$-division algebra $C$, and consequently $B . S$ is a compact set of generators of the $K$-algebra $A$, where $S$ is a basis of the $C$-algebra $A$. In sum, $A$ is an indiscrete, compactly generated, locally compact primitive $K$-algebra having minimal left ideals. Actually, every indiscrete, compactly generated, locally compact primitive $K$-algebra having minimal left ideals arises in this manner:

THEOREM 16. Let $K$ be an infinite topological field, and let $A$ be a locally compact primitive $K$-algebra having minimal left ideals. Then $A$ is compactly generated if and only if one of the following two possibilities holds:

$1^{\circ}$. A is topologically isomorphic to the locally compact K-algebra of all (continuous) linear operators on a finite-dimensional locally compact vector space over an indiscrete locally compact $K$-division algebra $D$.

$2^{\circ} . A$ is discrete and algebraically is isomorphic to the K-algebra of all linear operators on a finite-dimensional vector space over a $K$-division algebra $D$ satisfying $[D: K]<+\infty$.

Proof. To complete the proof that the condition is sufficient, we need merely observe that if $A$ is the algebra described in $2^{\circ}$, then $A$ is finite-dimensional over $K$ and hence is compactly generated. Necessity. Let $e$ be a minimal idempotent of $A$. Then $D=e A e$ is a locally compact $K$-division algebra whose topology is thus defined by an absolute value. If $D$ is indiscrete, the vector spaces $A e$ and $e A$ are finite-dimensional, and $1^{\circ}$ follows. We shall assume, therefore, that $D$ is discrete. If $V$ is a compact set that generates the $K$-vector space $A$, clearly $V e$ is a compact 
set that generates the $K$-vector space $A e$ and hence also the right $D$-vector space $A e$, and similarly $e V$ is a compact set that generates the $K$-vector space $e A$ and hence also the left $D$-vector space $e A$. Thus $A e$ and $e A$ are straight compactly generated locally compact vector spaces over the infinite discrete division ring $D$. By Jacobson's theorem [8, p. 77], the restriction of multiplication to $e A \times A e$ is a nondegenerate bilinear form. Suppose that $A e$ were indiscrete, whence $D$ would be an absolutely algebraic field of prime characteristic by Theorem 1 ; then $A e$ would be isomorphic to a subspace of the topological dual of the $D$-vector space $e A$ and hence would be countable by Theorem 13. But a countable locally compact group is discrete by Baire's theorem [4, Theorem 1, p. 110]. Hence $A e$ is necessarily discrete and consequently is a finite-dimensional vector space over $D$. Since the topology of $A_{L}$ is stronger than the compact-open topology, therefore, $A_{L}$ and hence $A$ are discrete. Thus $A$ is finite-dimensional over $K$, whence $D$ is also, and $2^{\circ}$ holds.

The same argument applies to locally compact primitive rings having minimal left ideals: If $e$ is a minimal idempotent of such a $\operatorname{ring} A$ such that $e A e$ is infinite and $A e$ and $e A$ are compactly generated $e A e$-vector spaces, then $A$ is topologically isomorphic to the ring of all linear operators on a finite-dimensional locally compact vector space over a locally compact division ring.

Theorem 16 shows that there is no analogue of Theorem 10 for algebras, that is, that in general a locally compact algebra is not the topological direct product of a compactly generated subalgebra and a discrete subalgebra. The following theorem improves Theorem 6 of [16].

THEOREM 17. Let $A$ be a locally compact primitive algebra having minimal left ideals over a topological field $K$. If any one of the following three conditions holds, then either $A$ is discrete, or $A$ contains an identity element and is finite-dimensional over its center.

$1^{\circ} . K$ is indiscrete.

$2^{\circ} . K$ is infinite but is not an absolutely algebraic field of prime characteristic.

$3^{\circ} . K$ is infinite, and $A$ is compactly generated.

Proof. Let $e$ be a minimal idempotent of $A$, and let $D=e A e$. If $D$ is indiscrete, then as in the proof of Theorem $16, A e$ is a finite-dimensional $D$-vector space, and the result follows. We shall assume, therefore, that $D$ is discrete. Then $K$ is also discrete as $\lambda \rightarrow \lambda e$ is continuous from $K$ into $D$; thus by hypothesis either $2^{\circ}$ or $3^{\circ}$ holds. To prove that $A$ is a straight $K$-algebra, let $a$ be a nonzero element of $A$. Then there exists $x \in e A$ such that $x a=a_{R}(x) \neq 0$. As $e A$ is a straight $D$-vector space, there exists a neighborhood $V$ of zero in $A$ such that $e V \cap D . x a=\{0\}$. Let $W$ be a neighborhood of zero in $A$ such that $x W \subseteq V$. If $w \in W \cap K$. a, then $w=\lambda a$ for some $\lambda \in K$, and hence $x w=(e x)(\lambda a)=(\lambda e)(x a) \in D \cdot x a$, and also $x w=e x w \in e V$; thus $\lambda e=0$, so $\lambda=0$ and hence $w=0$. Consequently, $A$ is straight. Therefore the conclusion follows if $2^{\circ}$ holds by Theorem 1 , and it follows also if $3^{\circ}$ holds by Theorem 16. 
We conclude with an example of an indiscrete straight locally compact dense algebra of linear operators on a discrete vector space. Let $K$ be a discrete infinite absolutely algebraic field of prime characteristic. Let $A$ be the algebra of all continuous linear operators on an indiscrete compactly generated straight locally compact $K$-vector space $E$ whose topological dual is total, and let $A$ be equipped with the compact-open topology. Then $A$ is an indiscrete straight locally compact dense algebra of linear operators by Theorem 15 , and moreover $A$ contains a projection $e$ on a one-dimensional subspace $K . c$ of $E$. As $A$ is dense and $K$ commutative, $\lambda \rightarrow \lambda e$ is an isomorphism from $K$ onto $e A e$; we shall identify $K$ with $e A e$ by means of this isomorphism. The algebraic isomorphism $u \rightarrow u(c)$ from the $K$-vector space $A e$ onto $E$ is clearly continuous; to show that it is open, let $U$ be a compact subset of $E$, let $V$ be a neighborhood of zero in $E$, and let $W=$ $\{u \in A: u(U) \subseteq V\}$. Then $e(U)$ is a compact subset of the discrete space $K . c$ and hence is finite; let $e(U)=\left\{\lambda_{1} c, \ldots, \lambda_{n} c\right\}$. Clearly $u \in A e \cap W$ if and only if $u(c) \in \lambda_{1}^{-1} V \cap \cdots \cap \lambda_{n}^{-1} V$. Thus $u \rightarrow u(c)$ is a topological isomorphism from $A e$ onto $E$; in particular, $A e$ is a compactly generated $K$-vector space. As in the proof of Theorem $16, e A$ is algebraically isomorphic to a subspace of $(A e)^{*}$, hence is countable, and therefore is discrete. Let $F$ be the discrete $K$-vector space $e A$, and let $B=A_{R}$, equipped with the topology for which the anti-isomorphism $a \rightarrow a_{R}$ from $A$ onto $B$ is a homeomorphism. Then $B$ is an indiscrete straight locally compact $K$-algebra, and $B$ is a dense algebra of linear operators on $F$. The topology $\mathscr{T}$ of $B$ is strictly stronger than the compact-open topology. Indeed, let $B^{\prime}$ be the algebra of all (continuous) linear operators on $F$, equipped with the compact-open topology $\mathscr{T}^{\prime}$. Then $B$ is dense in $B^{\prime}$ since $\mathscr{T}^{\prime}$ is also the topology of pointwise convergence. If the topology induced on $B$ by $\mathscr{T}^{\prime}$ were $\mathscr{T}$, then $B$ would be a complete dense subalgebra of $B^{\prime}$, so $B$ would be $B^{\prime}$ and hence $\mathscr{T}$ would be $\mathscr{T}^{\prime}$, which is impossible since by Theorem $15, \mathscr{T}^{\prime}$ is not locally compact.

\section{Commutative semisimple straight locally compact algebras over infinite discrete} fields. For each $\iota \in I$ let $C_{\iota}$ be a topological algebra with identity $1_{\iota}$ over a discrete field $K$, and let $H_{\iota}$ be an open subring of $C_{\iota}$ that contains $1_{\iota}$. We assume further that for each $\alpha \in K, \alpha .1_{\iota} \in H_{\iota}$ for all but finitely many $\iota \in I$. Then the subset $A$ of $\prod_{\iota \in I} C_{\iota}$, defined by

$$
A=\left\{\left(x_{\iota}\right) \in \prod_{\iota \in I} C_{\iota}: x_{\iota} \in H_{\iota} \text { for all but finitely many } \iota \in I\right\}
$$

is clearly a subalgebra of $\Pi C_{\imath}$; equipped with the topology for which the filter of neighborhoods of zero of the topological ring $\Pi H_{\iota}$ is a fundamental system of neighborhoods of zero, $A$ is easily seen to be a topological $K$-algebra, called the local direct product of $\left(C_{\iota}\right)_{i \in I}$ relative to $\left(H_{\iota}\right)_{\iota \in I}[7$, p. 7]. Any subalgebra of $A$ that contains $\prod H_{\iota}$, equipped with the induced topology, is called a local subdirect product of $\left(C_{\imath}\right)_{\iota \in I}$ relative to $\left(H_{\imath}\right)_{\iota \in I}$. It is easy to verify that $A$ is straight if and only if each $C_{\iota}$ is, and that if each $C_{\iota}$ is straight and $K$ countable, then $I$ is countable. 
If each $H_{\iota}$ is compact, then every local subdirect product is clearly locally compact. Also a local subdirect product $B$ is semisimple provided each of its projections $\operatorname{pr}_{\iota}(B)$ is semisimple [8, Proposition 1, p. 10]. In summary:

THEOREM 18. Let $K$ be an infinite discrete field, and for each $n \geqq 0$ let $C_{n}$ be $a$ straight commutative locally compact $K$-algebra with identity element $1_{n}$, and let $H_{n}$ be a compact open subring of $C_{n}$ that contains $1_{n}$. Assume further that for each $\alpha \in K$, $\alpha .1_{n} \in H_{n}$ for all but finitely many $n \geqq 0$. Then each local subdirect product $B$ of $\left(C_{n}\right)_{n \geqq 0}$ relative to $\left(H_{n}\right)_{n \geqq 0}$ such that each $\operatorname{pr}_{n}(B)$ is semisimple is a straight semisimple commutative locally compact $K$-algebra with identity.

Our structure theorem for straight semisimple commutative locally compact algebras over infinite discrete fields [Theorem 20] is that any such algebra is the topological direct product of a discrete algebra and a local subdirect product $B$ of $\left(C_{n}\right)_{n \geqq 0}$ relative to $\left(H_{n}\right)_{n \geqq 0}$, where each $C_{n}$ is algebraically the cartesian product of fields and topologically is discrete, where each $\operatorname{pr}_{n}(B)$ is an algebraically dense and hence semisimple subalgebra of $C_{n}$, and where each $H_{n}$ is a finite subfield of $C_{n}$ containing the identity element of $C_{n}$. However, to determine necessary and sufficient conditions for such an algebra to be, for example, compactly generated, it is desirable to direct attention to the fields of which $C_{n}$ is the cartesian product and the various subfields of given finite order of $C_{n}$ that contain the identity element.

For simplicity, let us assume that $K$ is an infinite subfield of the algebraic closure $\Omega$ of the field $Z_{p}$, that $C_{n}=\prod_{\gamma \in \Gamma_{n}} K_{\gamma}$, where each $K_{\gamma}$ is an extension field of $K$ all of whose elements algebraic over $Z_{p}$ belong to $\Omega$, and that the index sets $\Gamma_{n}$ are mutually disjoint, so that they form a partition of $\Gamma=\bigcup_{n \geqq 0} \Gamma_{n}$. If $H_{n}$ is a finite subfield of $C_{n}$ that contains the identity element $1_{n}=\left(1_{\gamma}\right)$ of $C_{n}$, then there is an isomorphism $\sigma_{n}$ from a finite subfield $F_{n}$ of $\Omega$ onto $H_{n}$; consequently for each $\gamma \in \Gamma_{n}, \sigma_{\gamma}=\operatorname{pr}_{\gamma} \circ \sigma_{n}$ (where $\mathrm{pr}_{\gamma}$ is the canonical projection from $C_{n}$ onto $K_{\gamma}$ ) is an isomorphism from $F_{n}$ into $K_{\gamma}$ as $1_{n} \in H_{n}$, and therefore $\sigma_{\gamma}$ is an automorphism of $F_{n}$ since $\Omega$ contains only one subfield having a prescribed finite number of elements; replacing the given isomorphism $\sigma_{n}$ from $F_{n}$ onto $H_{n}$ by $\sigma_{n} \sigma_{\beta}^{-1}$ for some $\beta \in \Gamma_{n}$, we may as well assume that one of the $\sigma_{\gamma}$ 's is the identity automorphism of $F_{n}$; moreover,

$$
H_{n}=\left\{\left(\sigma_{\gamma}(z)\right) \in \prod_{\gamma \in \Gamma_{n}} K_{\gamma}: z \in F_{n}\right\}
$$

Conversely, if $\left(\sigma_{\gamma}\right)_{y \in \Gamma_{n}}$ is a family of automorphisms of $F_{n}$ containing the identity automorphism, then $H_{n}$, defined as above, is a finite subfield of $C_{n}$ that contains its identity element and is canonically isomorphic to $F_{n}$. The requirement that for each $\alpha \in K, \alpha .1_{n} \in C_{n}$ for all but finitely many $n \geqq 0$ is clearly equivalent to the condition that for each $\alpha \in K$ there exists $m \geqq 0$ such that for all $n>m, \alpha \in F_{n}$ and $\sigma_{\gamma}(\alpha)=\alpha$ for all $\gamma \in \Gamma_{n}$. We are therefore led to the following definition:

Definition. Let $\Omega$ be the algebraic closure of the field $Z_{p}$ of integers modulo $p$, 
and let $K$ be an infinite subfield of $\Omega$. A family of $K$-ingredients consists of a family of fields $\left(K_{\gamma}\right)_{\gamma \in \Gamma}$ indexed by a set $\Gamma$, a sequence $\left(\Gamma_{n}\right)_{n \geqq 0}$ of subsets of $\Gamma$, a sequence $\left(F_{n}\right)_{n \geqq 0}$ of finite fields, and a family $\left(\sigma_{\gamma}\right)_{\gamma \in \Gamma}$ of mappings such that the following conditions hold:

$1^{\circ}$. Each $K_{\gamma}$ is an extension of $K$, and the field of elements of $K_{y}$ algebraic over $Z_{p}$ is a subfield of $\Omega$.

$2^{\circ}$. $\left(\Gamma_{n}\right)_{n \geqq 0}$ is a partition of $\Gamma$.

$3^{\circ}$. For each $n \geqq 0,\left(K_{\gamma}\right)_{\gamma \in \Gamma_{n}}$ is a family of field extensions of $F_{n}$, and $\left(\sigma_{\gamma}\right)_{\gamma \in \Gamma_{n}}$ is a family of automorphisms of $F_{n}$ that contains the identity automorphism.

$4^{\circ}$. For each $\alpha \in K$ there exists $m \geqq 0$ such that for all $n>m, \alpha \in F_{n}$ and $\sigma_{\gamma}(\alpha)=\alpha$ for all $\gamma \in \Gamma_{n}$.

Let $\left(K_{\gamma}\right)_{\gamma \in \Gamma},\left(\Gamma_{n}\right)_{n \geqq 0},\left(F_{n}\right)_{n \geqq 0},\left(\sigma_{\gamma}\right)_{\gamma \in \Gamma}$ be a family of $K$-ingredients. For each $n \geqq 0$ let $C_{n}$ be the $K$-algebra $\prod_{\gamma \in \Gamma_{n}} K_{\gamma}$, equipped with the discrete topology, and let $H_{n}$ be the subfield $\left\{\left(\sigma_{y}(z)\right) \in C_{n}: z \in F_{n}\right\}$ of $C_{n}$. The full algebra defined by $\left(K_{\gamma}\right)_{\gamma \in \Gamma},\left(\Gamma_{n}\right)_{n \geqq 0},\left(F_{n}\right)_{n \geqq 0},\left(\sigma_{\gamma}\right)_{\gamma \in \Gamma}$ is the local direct product $A$ of $\left(C_{n}\right)_{n \geqq 0}$ relative to $\left(H_{n}\right)_{n \geqq 0}$, and the ring defined by that family is the ring $V$ defined by

$$
\begin{aligned}
V=\prod_{n \geqq 0} H_{n}=\left\{\left(x_{\gamma}\right) \in \prod_{\gamma \in \Gamma} K_{\gamma}: \text { there exists }\left(z_{n}\right) \in \prod_{n \geqq 0} F_{n}\right. \\
\\
\left.\quad \text { such that for every } n \geqq 0, x_{\gamma}=\sigma_{\gamma}\left(z_{n}\right) \text { for all } \gamma \in \Gamma_{n}\right\}
\end{aligned}
$$

(we identify canonically the $K$-algebra $\prod_{n \geqq 0} C_{n}$ with the $K$-algebra $\prod_{\gamma \in \Gamma} K_{\gamma}$ ). Each local subdirect product $B$ of $\left(C_{n}\right)_{n \geqq 0}$ relative to $\left(H_{n}\right)_{n \geqq 0}$ that satisfies the following density property is called an algebra defined by that family: For each $\left(a_{\gamma}\right) \in A$ and each finite subset $\Delta$ of $\Gamma$, there exists $\left(b_{\gamma}\right) \in B$ such that $b_{\gamma}=a_{\gamma}$ for all $\gamma \in \Delta$.

Clearly if each $\Gamma_{n}$ is finite, the only algebra defined by this family of $K$-ingredients is the full algebra $A$. For future reference, let $e_{k}$ be the idempotent of $V$ whose $\gamma$ th component is 1 or 0 according as $\gamma \in \Gamma_{k}$ or $\gamma \notin \Gamma_{k}$. Then $V\left(1-\sum_{k=0}^{m} e_{k}\right)=$ $\prod_{n \geqq 0} H_{n}^{\prime}$ where $H_{n}^{\prime}=\{0\}$ if $n \leqq m$ and $H_{n}^{\prime}=H_{n}$ if $n>m$; consequently the sequence $\left(V\left(1-\sum_{k=0}^{m} e_{k}\right)\right)_{m \geqq 0}$ is a fundamental system of neighborhoods of zero in any algebra defined by that family of $K$-ingredients.

THEOREM 19. Let $K$ be an infinite subfield of the algebraic closure $\Omega$ of $Z_{p}$, equipped with the discrete topology. Let $A$ be a commutative semisimple indiscrete straight locally compact $K$-algebra. If $B$ is the ideal of $A$ generated by a compact open subring $V$, then there is a topological isomorphism $\Phi$ from $B$ onto a locally compact $K$-algebra $B^{\prime}$ defined by a family of $K$-ingredients that takes $V$ onto the ring $V^{\prime}$ defined by that family.

Proof. Since $B$ is an ideal of $A, B$ is semisimple [8, Theorem 1, p. 10], and $B$ is, of course, commutative and locally compact. We first observe that by a theorem of Kaplansky, the intersection of all the closed regular maximal ideals of $B$ is $\{0\}$. 
Indeed, this follows from [10, Theorem 12] if $B$ has an identity element, since $K$ has prime characteristic and $B$ is totally disconnected (Theorems 1 and 2). If $B$ lacks an identity element, we may apply Kaplansky's theorem to $B^{+}$, the $K$-algebra containing $B$ as an ideal that is the vector space direct sum of $B$ and the onedimensional subspace generated by an identity element $e$. To show that the $V$ topology on $B^{+}$is compatible with multiplication, it suffices to show that $x \rightarrow x(b+\beta e)$ is continuous at zero, where $b \in B, \beta \in K^{*}$; but if $U$ is an open subgroup of $B$ and if $W$ is an open subgroup of $U$ such that $W b \subseteq U$, then $\left(W \cap \beta^{-1} W\right)(b+\beta e) \subseteq U$. Also, $B^{+}$is straight since $V \cap K . b=\{0\}$ if $b \notin B$. To show that if $\mathfrak{m}$ is a maximal ideal of $B^{+}$other than $B$, then $\mathfrak{m} \cap B$ is a maximal ideal of $B$, it suffices to show that the canonical monomorphism $h: x+(\mathfrak{m} \cap B) \rightarrow$ $x+\mathfrak{m}$ from $B /(\mathfrak{m} \cap B)$ into $B^{+} / \mathfrak{m}$ is actually surjective, for then $B /(\mathfrak{m} \cap B)$ is a field. As $\mathfrak{m}$ is not contained in $B$, there exist $\alpha \neq 0$ and $c \in B$ such that $c+\alpha e \in \mathfrak{m}$; consequently, for any $b+\beta e \in B^{+}$,

$$
\begin{aligned}
h\left(\left(b-\alpha^{-1} \beta c\right)+(\mathfrak{m} \cap A)\right) & =\left(b-\alpha^{-1} \beta c\right)+\mathfrak{m} \\
& =\left(b-\alpha^{-1} \beta c\right)+\alpha^{-1} \beta(c+\alpha e)+\mathfrak{m} \\
& =(b+\beta e)+\mathfrak{m} .
\end{aligned}
$$

Now $B^{+}$is semisimple (cf. [8, p. 11]); therefore by Kaplansky's theorem the intersection of the closed maximal ideals of $B^{+}$is $\{0\}$; consequently by the preceding, the intersection of the closed regular maximal ideals of $B$ is $\{0\}$.

Next we shall prove that a closed regular maximal ideal $\mathfrak{m}$ of $B$ is open. Equipped with its quotient topology, $B / \mathfrak{m}$ is a locally compact ring that algebraically is a field; hence $B / \mathfrak{m}$ is a locally compact field [12, Theorem 3], [10, Theorems 7-9]. Let $K^{\prime}$ be the image of $K$ in $B / \mathfrak{m}$ under the canonical monomorphism $\lambda \rightarrow \lambda e+\mathfrak{m}$ where $e+\mathfrak{m}$ is the identity element of $B / \mathfrak{m}$. If $a$ is a nonzero element of $K^{\prime}$, then every power of $a$ belongs to $P[a]^{*}$, where $P$ is the prime subfield of $K^{\prime}$. But $P[a]^{*}$ is finite as $K$ is an absolutely algebraic field of prime characteristic, and consequently $a^{n} \nrightarrow 0$. As $K^{\prime}$ is an infinite discrete subfield of the locally compact field $B / \mathfrak{m}$, therefore, $B / \mathfrak{m}$ is discrete. Thus $\mathfrak{m}$ is open.

We shall show next that if $\mathfrak{m}$ is a closed regular maximal ideal of $B$, then $\mathfrak{m} \cap V$ is an open regular maximal ideal of $V$. Indeed, as $\mathfrak{m}$ is a regular ideal of the ideal $B$, $\mathfrak{m}$ is an ideal of $A$ and consequently $\mathfrak{m} \cap V$ is a proper ideal of $V$ as the ideal generated by $V$ is $B$. Also, $\mathfrak{m} \cap V$ is open by what we have just proved, so $V /(\mathfrak{m} \cap V)$ is discrete and compact and hence is a finite ring. But $V /(\mathfrak{m} \cap V)$ is isomorphic to the subring $(V+\mathfrak{m}) / \mathfrak{m}$ of the field $B / \mathfrak{m}$ and hence is a finite commutative ring having no proper zero divisors. Consequently, $V /(\mathfrak{m} \cap V)$ is a finite field, so $\mathfrak{m} \cap V$ is an open regular maximal ideal of $V$.

Let $\left(\mathfrak{m}_{\gamma}\right)_{\gamma \in \Gamma}$ be a family of distinct closed regular maximal ideals of $B$ such that $\bigcap_{\gamma \in \Gamma} \mathfrak{m}_{\gamma}=\{0\}$ (for example, by the preceding we may choose $\left(\mathfrak{m}_{\gamma}\right)_{y \in \Gamma}$ to be the family of all closed regular maximal ideals of $B$ ). Let $\left(\Gamma_{n}\right)_{n \in S}$ be the partition of $\Gamma$ determined by the equivalence relation $R$ on $\Gamma$ satisfying $\beta R \gamma$ if and only if $\mathfrak{m}_{\beta} \cap V$ 
$=\mathfrak{m}_{\gamma} \cap V$. (We shall shortly see that $S$ is denumerable and hence may be taken to be the set of natural numbers.) For each $\gamma \in \Gamma$ let $\phi_{\gamma}$ be the canonical epimorphism from $B$ onto $B / \mathfrak{m}_{\gamma}$, let $g_{\gamma}$ be a $K$-isomorphism from $B / \mathfrak{m}_{y}$ onto an extension field $K_{y}$ of $K$ all of whose elements algebraic over $Z_{p}$ belong to $\Omega$, and let $h_{\gamma}$ be the canonical monomorphism $x+\left(\mathfrak{m}_{\gamma} \cap V\right) \rightarrow x+\mathfrak{m}_{\gamma}$ from $V /\left(\mathfrak{m}_{\gamma} \cap V\right)$ onto $\left(V+\mathfrak{m}_{\gamma}\right) / \mathfrak{m}_{\gamma}$ $=\phi_{y}(V)$. For each $n \in S$ let $\beta_{n}$ be an element of $\Gamma_{n}$, and let $F_{n}=g_{\beta_{n}}\left(\phi_{\beta_{n}}(V)\right)$. For each $\gamma \in \Gamma_{n}, \phi_{\gamma}(V)$ is isomorphic to $V /\left(\mathfrak{m}_{\gamma} \cap V\right)=V /\left(\mathfrak{m}_{\beta_{n}} \cap V\right)$ and hence to $\phi_{\beta_{n}}(V)$; we saw earlier that $V /\left(\mathfrak{m}_{\gamma} \cap V\right)$ is a finite field; therefore $g_{\gamma} \phi_{\gamma}(V)$ is a finite subfield of $K_{\gamma}$ having the same number of elements as $F_{n}$, and hence $g_{\gamma} \phi_{\gamma}(V)=F_{n}$ as a field contains at most one subfield having a prescribed finite number of elements. For each $\gamma \in \Gamma_{n}$ let

$$
\sigma_{\gamma}=g_{\gamma} \circ h_{\gamma} \circ h_{\beta_{n}}^{-1} \circ\left(g_{\beta_{n}}^{-1} \mid F_{n}\right) \text {. }
$$

Then $\sigma_{y}$ is an automorphism of $F_{n}$, and for each $x \in V$,

$$
g_{\gamma} \phi_{\gamma}(x)=\sigma_{\gamma}\left(g_{\beta_{n}}\left(\phi_{\beta_{n}}(x)\right)\right)
$$

for all $\gamma \in \Gamma_{n}$. Moreover, $\sigma_{\beta_{n}}$ is the identity automorphism of $F_{n}$.

Let $\Psi$ be the mapping from $V$ into $\prod_{n \in S} F_{n}$ defined by

$$
\Psi(x)=\left(g_{\beta_{n}}\left(\phi_{\beta_{n}}(x)\right)\right)_{n \in S} .
$$

The kernel of the restriction to $V$ of $g_{\beta_{n}} \phi_{\beta_{n}}$ is the open regular maximal ideal $\mathfrak{m}_{\beta_{n}} \cap V$ of $V$, so $\Psi$ is continuous from $V$ into $\prod_{n \in S} F_{n}$, where each $F_{n}$ has the discrete topology. The kernel of $\Psi$ is

$$
\bigcap_{n \in S}\left(\mathfrak{m}_{\beta_{n}} \cap V\right)=\bigcap_{\gamma \in \Gamma}\left(\mathfrak{m}_{\gamma} \cap V\right)=\{0\} .
$$

Also, as $\left(\mathfrak{m}_{\beta_{n}} \cap V\right)_{n \in S}$ is a family of distinct regular maximal ideals of $V, \Psi(V)$ is dense in $\prod_{n \in S} F_{n}$ by the Chinese Remainder Theorem [14, Exercise 35.25(c), p. 393]. Therefore $\Psi(V)$ is a compact dense subset of $\prod_{n \in S} F_{n}$ and hence is all of $\prod_{n \in S} F_{n}$. In particular, $V$ has an identity element; also as $V$ is metrizable, $S$ is countable, so we may assume that $S$ is the set of natural numbers.

To show that $\left(K_{\gamma}\right)_{y \in \Gamma},\left(\Gamma_{n}\right)_{n \geqq 0},\left(F_{n}\right)_{n \geqq 0},\left(\sigma_{\gamma}\right)_{\gamma \in \Gamma}$ is a family of $K$-ingredients, it remains for us to show that $4^{\circ}$ of the Definition holds. Let $\alpha \in K^{*}$. As $\Psi$ is a topological isomorphism, there exists $m \geqq 0$ such that $\Psi\left(V \cap \alpha^{-1} V\right) \supseteq \prod_{n \geqq 0} L_{n}$ where $L_{n}=\{0\}$ if $n \leqq m$ and $L_{n}=F_{n}$ if $n>m$. Consequently there exists $w \in V \cap \alpha^{-1} V$ such that $\Psi(w)=\left(\varepsilon_{n}\right)$, where $\varepsilon_{n}=0$ if $n \leqq m$ and $\varepsilon_{n}=1$ if $n>m$. Let $n>m$. Then $g_{\beta_{n}}\left(\phi_{\beta_{n}}(w)\right)=1$, so

$$
\alpha=g_{\beta_{n}} \phi_{\beta_{n}}(\alpha w) \in g_{\beta_{n}} \phi_{\beta_{n}}(V)=F_{n} .
$$

Also if $\gamma \in \Gamma_{n}$, then by (1)

$$
\begin{aligned}
\sigma_{\gamma}(\alpha) & =\sigma_{\gamma}\left(g_{\beta_{n}}\left(\phi_{\beta_{n}}(\alpha w)\right)\right)=g_{\gamma} \phi_{\gamma}(\alpha w) \\
& =\alpha g_{\gamma} \phi_{\gamma}(w)=\alpha \sigma_{\gamma}\left(g_{\beta_{n}}\left(\phi_{\beta_{n}}(w)\right)\right) \\
& =\alpha \sigma_{\gamma}(1)=\alpha .
\end{aligned}
$$


Let $A^{\prime}$ and $V^{\prime}$ be respectively the full $K$-algebra and ring determined by the family of $K$-ingredients $\left(K_{\gamma}\right)_{y \in \Gamma},\left(\Gamma_{n}\right)_{n \geqq 0},\left(F_{n}\right)_{n \geqq 0},\left(\sigma_{\gamma}\right)_{y \in \Gamma}$, and let

$$
\Phi(x)=\left(g_{\gamma} \phi_{\gamma}(x)\right)_{\gamma \in \Gamma}
$$

for all $x \in B$. By (1), $\Phi(V) \subseteq V^{\prime}$. Conversely, let $\left(y_{\gamma}\right) \in V^{\prime}$, and let $\left(z_{n}\right) \in \prod_{n \geqq 0} F_{n}$ be such that $\sigma_{y}\left(z_{n}\right)=y_{\gamma}$ for all $\gamma \in \Gamma_{n}$ and all $n \geqq 0$. Then by the preceding, there exists $x \in V$ such that $\Psi(x)=\left(z_{n}\right)$, whence for each $n \geqq 0$ and each $\gamma \in \Gamma_{n}$,

$$
g_{\gamma} \phi_{\gamma}(x)=\sigma_{\gamma}\left(g_{\beta_{n}}\left(\phi_{\beta_{n}}(x)\right)\right)=\sigma_{\gamma}\left(z_{n}\right)=y_{\gamma}
$$

by (1). Thus $\Phi(x)=\left(y_{y}\right)$; hence $\Phi(V)=V^{\prime}$.

To show that $\Phi(B) \subseteq A^{\prime}$, let $a \in B$. As $z \rightarrow z a$ is continuous, there is a neighborhood $W$ of zero in $V$ such that $W a \subseteq V$. As before, there exists $m \geqq 0$ and $w \in W$ such that $g_{\beta_{n}} \phi_{\beta_{n}}(w)$ is 0 or 1 according as $n \leqq m$ or $n>m$. For each $n \geqq 0$ let $z_{n}=g_{\beta_{n}} \phi_{\beta_{n}}(w a) \in F_{n}$. If $n>m$ and if $\gamma \in \Gamma_{n}$, then by (1), as $w$ and $w a$ belong to $V$,

$$
\begin{aligned}
\sigma_{\gamma}\left(z_{n}\right) & =g_{\gamma} \phi_{\gamma}(w a)=g_{\gamma} \phi_{\gamma}(w) \cdot g_{\gamma} \phi_{\gamma}(a) \\
& =\sigma_{\gamma}\left(g_{\beta_{n}}\left(\phi_{\beta_{n}}(w)\right)\right) \cdot g_{\gamma} \phi_{\gamma}(a) \\
& =\sigma_{\gamma}(1) \cdot g_{\gamma} \phi_{\gamma}(a)=g_{\gamma} \phi_{\gamma}(a) .
\end{aligned}
$$

Thus $\Phi(a) \in A^{\prime}$. By the Chinese Remainder Theorem, if $\left(a_{\gamma}\right) \in A^{\prime}$ and if $\Delta$ is a finite subset of $\Gamma$, there exists $\left(b_{\gamma}\right) \in \Phi(B)$ such that $b_{\gamma}=a_{\gamma}$ for all $\gamma \in \Delta$. Thus as the topology of $B$ is the $V$-topology, $\Phi$ is a topological isomorphism from $B$ onto a locally compact $K$-algebra defined by a family of $K$-ingredients.

THEOREM 20. Let $K$ be an infinite subfield of the algebraic closure of $Z_{p}$, equipped with the discrete topology. A commutative semisimple indiscrete straight locally compact $K$-algebra $A$ is the topological direct product of a discrete commutative semisimple $K$-algebra and a $K$-algebra topologically isomorphic to a locally compact algebra defined by a family of $K$-ingredients.

Proof. As $A$ is totally disconnected (Theorem 2), $A$ contains a compact open subring $V$ [10, Lemma 4]. Let $B$ be the ideal generated by $V$. By Theorem $19, B$ is topologically isomorphic to a locally compact algebra defined by a family of $K$-ingredients. In particular, $B$ has an identity element $e$, and hence $A$ is the direct product of the ideals $B$ and $C=\{x-x e: x \in A\}$. As $C \cap V=\{0\}, C$ is discrete and the projection on $C$ along $B$ is continuous; hence $A$ is the topological direct product of $B$ and $C$. As $C$ is an ideal of $A, C$ is semisimple [8, Theorem 1, p. 10].

Certain natural questions present themselves concerning the family of $K$ ingredients $\left(K_{\gamma}\right)_{\gamma \in \Gamma},\left(\Gamma_{n}\right)_{n \geqq 0},\left(F_{n}\right)_{n \geqq 0},\left(\sigma_{\gamma}\right)_{\gamma \in \Gamma}$ occurring in the proof of Theorem 19, namely: (1) When does $\Phi(B)$ contain the outer direct sum $\bigoplus_{\gamma \in \Gamma} K_{\gamma}$ of $\left(K_{\gamma}\right)_{\gamma \in \Gamma}$ ? (2) When is each $\Gamma_{n}$ finite? (3) What special properties must the family possess for $B$ to be compactly generated? (4) What is the relationship between two families of $K$-ingredients defining isomorphic locally compact algebras? 
Minimal ideals play an important role in answering these questions. We recall that the socle of a commutative ring is the sum of all its minimal ideals.

LEMMA 6. Let $A$ be a commutative ring having no nonzero nilpotent ideals.

$1^{\circ}$. The minimal ideals of $A$ are precisely those ideals that are subfields of $A$.

$2^{\circ}$. If $\mathfrak{m}$ is the annihilator of an ideal $\mathfrak{a}$, then $\mathfrak{a}$ is minimal if and only if $\mathfrak{m}$ is a regular maximal ideal, in which case $A$ is the direct product of $\mathfrak{a}$ and $\mathfrak{m}$.

$3^{\circ}$. If $A$ is the direct product of ideals $B$ and $C$ and if $\mathfrak{a}$ is a minimal ideal of $A$, then $\mathfrak{a}$ is either a minimal ideal of $B$ or a minimal ideal of $C$.

Proof. $1^{\circ}$ is a consequence of [8, Proposition 1, p. 57; Proposition 1, p. 65]. Consequently, if $\mathfrak{a}$ is a minimal ideal and if $e$ is its identity element, the annihilator $\mathfrak{m}$ of $\mathfrak{a}$ is $\{x-x e: x \in A\}$; therefore $A$ is the direct product of $\mathfrak{m}$ and $\mathfrak{a}$, so $A / \mathfrak{m}$ is isomorphic to the field $\mathfrak{a}$ and hence $\mathfrak{m}$ is a regular maximal ideal. Conversely, if the annihilator $\mathfrak{m}$ of $\mathfrak{a}$ is a regular maximal ideal, then $\mathfrak{m} \cap \mathfrak{a}=\{0\}$ as $A$ has no nonzero nilpotent ideals, and $A=\mathfrak{m}+\mathfrak{a}$; thus $A$ is the direct product of $\mathfrak{m}$ and $\mathfrak{a}$, and consequently $\mathfrak{a}$ is minimal since it is isomorphic to the field $A / \mathfrak{m}$. To prove $3^{\circ}$, let $e$ be the identity element of a minimal ideal $a$, and let $e=e_{1}+e_{2}$ where $e_{1} \in B, e_{2} \in C$. Then $e_{1} e_{2}=0$, so $e_{1}=e_{1} e \in \mathfrak{a}, e_{2}=e_{2} e \in \mathfrak{a}$, and $e_{1}^{2}+e_{2}^{2}=\left(e_{1}+e_{2}\right)^{2}$ $=e_{1}+e_{2}$ and thus $e_{1}$ and $e_{2}$ are idempotents of $\mathfrak{a}$; as $\mathfrak{a}$ is a field, one of $e_{1}, e_{2}$ is 0 and the other is $e$; hence either $\mathfrak{a} \subseteq B$ or $\mathfrak{a} \subseteq C$. As $\mathfrak{a}$ is a field, $\mathfrak{a}$ is clearly a minimal ideal of any subring of $A$ that contains it.

LEMMA 7. Let $A$ be a commutative ring, let $\left(\mathfrak{m}_{\gamma}\right)_{y \in \Gamma}$ be a family of distinct regular maximal ideals of $A$ such that $\bigcap_{y \in \Gamma} \mathfrak{m}_{y}=\{0\}$, and let $A^{\prime}$ be the image of $A$ under the monomorphism $\Phi: x \rightarrow\left(x+\mathfrak{m}_{y}\right)$ from $A$ into $\prod_{\gamma \in \Gamma}\left(A / \mathfrak{m}_{\gamma}\right)$. The following statements are equivalent:

$1^{\circ}$. $A^{\prime} \supseteq \bigoplus_{\gamma \in \Gamma}\left(A / \mathfrak{m}_{\gamma}\right)$.

$2^{\circ}$. Each $\mathfrak{m}_{y}$ is the annihilator of a (necessarily minimal) ideal.

$3^{\circ}$. If $\Delta \subset \Gamma$, then $\bigcap_{\gamma \in \Delta} \mathfrak{m}_{\gamma} \neq\{0\}$.

Moreover, if these conditions hold, the socle $S$ of $A^{\prime}$ is $\bigoplus_{\gamma \in \Gamma}\left(A / \mathfrak{m}_{\gamma}\right)$.

Proof. By hypothesis, $A$ is semisimple and hence has no nonzero nilpotent ideals. Assume that $1^{\circ}$ holds. Then for each $\beta \in \Gamma$ there exists a nonzero $y_{\beta} \in A$ such that $y_{\beta} \in \mathfrak{m}_{y}$ for all $\gamma \neq \beta$. If $x \in \mathfrak{m}_{\beta}$, then $x y_{\beta} \in \bigcap_{\gamma \in \Gamma} \mathfrak{m}_{\gamma}=\{0\}$; hence $\mathfrak{m}_{\beta}$ is contained in the annihilator $\mathfrak{m}^{\prime}$ of the ideal generated by $y_{\beta}$; as $A$ is semisimple, $\mathfrak{m}^{\prime}$ is a proper ideal, and therefore $\mathfrak{m}_{\beta}=\mathfrak{m}^{\prime}$. Thus $1^{\circ}$ implies $2^{\circ}$.

To show that $2^{\circ}$ implies $3^{\circ}$, for each $\gamma \in \Gamma$ let $e_{\gamma}$ be the identity element of the (minimal) ideal annihilated by $\mathfrak{m}_{\beta}$. If $\beta \in \Gamma$, then $e_{\beta} e_{\gamma}=0$ for all $\gamma \neq \beta$ as $A e_{\beta} \cap A e_{\gamma}=\{0\}$, so $e_{\beta} \in \bigcap_{\gamma \neq \beta} \mathfrak{m}_{\gamma}$.

For each $\beta \in \Gamma$ let $e_{\beta}^{\prime}$ be the idempotent of $\oplus_{\gamma \in \Gamma}\left(A / \mathfrak{m}_{\gamma}\right)$ whose $\gamma$ th component is 1 or 0 according as $\gamma=\beta$ or $\gamma \neq \beta$. If $1^{\circ}$ does not hold, then $e_{\beta}^{\prime} \notin A^{\prime}$ for some $\beta \in \Gamma$; hence for every $x \in A$, if $x \notin \mathfrak{m}_{\beta}$, then $x \notin \mathfrak{m}_{y}$ for some $\gamma \neq \beta$; consequently, if $x \in \bigcap_{\gamma \neq \beta} \mathfrak{m}_{\gamma}$, then $x \in \bigcap_{\gamma \in \Gamma} \mathfrak{m}_{\gamma}=\{0\}$, so $\bigcap_{\gamma \neq \beta} \mathfrak{m}_{\gamma}=\{0\}$, and $3^{\circ}$ does not hold. 
Assume finally that $1^{\circ}-3^{\circ}$ hold. The minimal idempotents of $A^{\prime}$ are then clearly the idempotents $e_{\beta}^{\prime}$ where $\beta \in \Gamma$, so $S=\bigoplus_{\gamma \in \Gamma}\left(A / \mathfrak{m}_{y}\right)$.

LeMma 8. Let $S$ be the socle of a nonzero commutative ring $A$ that contains no nonzero nilpotent ideals. The following statements are equivalent:

$1^{\circ}$. Every nonzero ideal of $A$ contains a minimal ideal.

$2^{\circ}$. The annihilator $S^{\prime}$ of $S$ is $\{0\}$.

$3^{\circ}$. There is a family $\left(K_{\gamma}\right)_{y \in \Gamma}$ of fields such that $A$ is isomorphic to a subring $A^{\prime}$ of $\prod_{\gamma \in \Gamma} K_{\gamma}$ that contains $\bigoplus_{\gamma \in \Gamma} K_{\gamma}$.

Proof. To show that $1^{\circ}$ implies $2^{\circ}$, let $x$ be a nonzero element of $A$. By $1^{\circ}, A x$ contains a minimal ideal; let $e$ be its identity element. Then $a x=e$ for some $a \in A$, so $x e \neq 0$ as $a x e=e$. Therefore $x$ does not belong to the annihilator of the minimal ideal $A e$. Consequently, $2^{\circ}$ holds as $S^{\prime}$ is the intersection of the annihilators of the minimal ideals of $A$.

If $\left(\mathfrak{m}_{y}\right)_{y \in \Gamma}$ is the set of annihilators of the distinct minimal ideals of $A$, then $S^{\prime}=\bigcap_{\gamma \in \Gamma} \mathfrak{m}_{y}$ and each $\mathfrak{m}_{y}$ is a regular maximal ideal of $A$ by Lemma 6 ; consequently, $2^{\circ}$ implies $3^{\circ}$ by Lemma 7 . Finally, to show that $3^{\circ}$ implies $1^{\circ}$, let $x=\left(\lambda_{\gamma}\right)$ be a nonzero element of $A^{\prime}$, and let $\beta$ be such that $\lambda_{\beta} \neq 0$. Then $y=\left(\mu_{\gamma}\right) \in \oplus K_{\gamma}$, where $\mu_{\beta}=\lambda_{\beta}^{-1}$ and $\mu_{\gamma}=0$ if $\gamma \neq \beta$; clearly $A y x$ is an ideal isomorphic to $K_{\beta}$ that is contained in $A x$.

THEOREM 21. Let $K$ be an infinite subfield of the algebraic closure of $Z_{p}$, equipped with the discrete topology. Let $B$ be a commutative semisimple indiscrete straight locally compact K-algebra that contains a compact open subring $V$ such that the ideal generated by $V$ is $B$ itself, and let $S$ be the socle of $B$. Then $B$ is topologically isomorphic to a locally compact $K$-algebra $B^{\prime}$ defined by a family of $K$-ingredients $\left(K_{\gamma}\right)_{y \in \Gamma},\left(\Gamma_{n}\right)_{n \geqq 0},\left(F_{n}\right)_{n \geqq 0},\left(\sigma_{\gamma}\right)_{y \in \Gamma}$ such that $B^{\prime} \supseteq \bigoplus_{\gamma \in \Gamma} K_{\gamma}$ if and only if the annihilator $S^{\prime}$ of $S$ is $\{0\}$.

Proof. The condition is necessary by Lemma 8 .

Sufficiency. Let $\left(\mathfrak{m}_{\gamma}\right)_{y \in \Gamma}$ be the annihilators of the distinct minimal ideals of $A$. By hypothesis, $\bigcap_{\gamma \in \Gamma} \mathfrak{m}_{\gamma}=\{0\}$. By Lemma 6 , each $\mathfrak{m}_{\gamma}$ is a regular maximal ideal. Also, $\mathfrak{m}_{y}=\left\{x \in A: x e_{y}=0\right\}$ where $e_{\gamma}$ is the identity element of the minimal ideal annihilated by $\mathfrak{m}_{\gamma}$, so $\mathfrak{m}_{y}$ is closed. Consequently $\left(\mathfrak{m}_{\gamma}\right)_{\gamma \in \Gamma}$ satisfies the conditions given in the proof of Theorem 19, and the result follows by that theorem and Lemma 7.

THEOREM 22. Let $K$ be an infinite subfield of the algebraic closure of $Z_{p}$, equipped with the discrete topology. Let $A$ be a commutative semisimple indiscrete straight locally compact K-algebra, and let $S$ be the socle of $A$. Then $A$ is the topological direct product of a discrete commutative semisimple $K$-algebra and a $K$-algebra topologically isomorphic to a locally compact algebra $B^{\prime}$ defined by a family of $K$-ingredients $\left(K_{\gamma}\right)_{y \in \Gamma},\left(\Gamma_{n}\right)_{n \geqq 0},\left(F_{n}\right)_{n \geqq 0},\left(\sigma_{\gamma}\right)_{y \in \Gamma}$ such that $B^{\prime} \supseteq \bigoplus_{\gamma \in \Gamma} K_{\gamma}$ if and only if the annihilator of the annihilator of $S$ is an open ideal. 
Proof. Let $S^{\prime}$ be the annihilator of $S, S^{\prime \prime}$ the annihilator of $S^{\prime}$.

Necessity. Let $A$ be the topological direct product of an ideal $B$ topologically isomorphic to $B^{\prime}$ and a discrete ideal $C$. As $B$ has an identity element, every ideal of $B$ is an ideal of $A$, so the socle of $B$ is $S \cap B$. Let $z=x+y \in S^{\prime}$, where $x \in B$, $y \in C$. Then for all $s \in S \cap B, 0=z s=x s+y s=x s$, so $x$ belongs to the annihilator in $B$ of the socle of $B$, and hence $x=0$ by Theorem 21. Thus $S^{\prime} \subseteq C$, so $S^{\prime \prime} \supseteq B$, which is open as $C$ and hence also $A / B$ are discrete.

Sufficiency. As $S^{\prime \prime}$ is an open ideal of $A, S^{\prime \prime}$ is semisimple, indiscrete, and locally compact. By Theorem $20, S^{\prime \prime}$ is the topological direct product of a discrete ideal $C_{1}$ and an indiscrete ideal $B$ containing a compact open subring $V$ that possesses the identity element $e$ of $B$. As $C_{1}$ and hence $S^{\prime \prime} \mid B$ are discrete, $B$ is open in $S^{\prime \prime}$ and hence in $A$. As in the proof of Theorem 20, $A$ is the topological direct product of $B$ and $C=\{x-x e: x \in A\}$, and $C$ is discrete. By Theorem 21, it remains for us to show that the annihilator $S_{0}^{\prime}$ in $B$ of the socle $S_{0}$ of $B$ is $\{0\}$. As $B$ has an identity element, every ideal of $B$ is an ideal of $A$, so $S_{0}=S \cap B$. If $x \in S_{0}^{\prime}$ and if $\mathfrak{a}$ is a minimal ideal of $A$, then by Lemma 6 either $\mathfrak{a} \subseteq S_{0}$, in which case $x \mathfrak{a}=\{0\}$, or $\mathfrak{a} \subseteq C$, in which case $x \mathfrak{a} \subseteq B C=\{0\}$. Therefore $S_{0}^{\prime}=S^{\prime} \cap B \subseteq S^{\prime} \cap S^{\prime \prime}=\{0\}$.

THEOREM 23. Let $K$ be an infinite subfield of the algebraic closure of $Z_{p}$, equipped with the discrete topology. Let $A$ be a commutative semisimple indiscrete straight locally compact $K$-algebra that contains a compact open subring $V$ such that the ideal generated by $V$ is $A$ itself, and let $S$ be the socle of $A$. Then $A$ is topologically isomorphic to the locally compact full $K$-algebra $A^{\prime}$ defined by a family of $K$-ingredients $\left(K_{\gamma}\right)_{\gamma \in \Gamma},\left(\Gamma_{n}\right)_{n \geqq 0},\left(F_{n}\right)_{n \geqq 0},\left(\sigma_{\gamma}\right)_{y \in \Gamma}$ where each $\Gamma_{n}$ is finite if and only if $S$ is dense in $A$.

Proof. If the socle of $A$ is dense, clearly its annihilator is $\{0\}$. Hence by Theorem 21 it suffices to prove that if $B$ is a locally compact algebra defined by a family of $K$-ingredients $\left(K_{\gamma}\right)_{y \in \Gamma},\left(\Gamma_{n}\right)_{n \geqq 0},\left(F_{n}\right)_{n \geqq 0},\left(\sigma_{\gamma}\right)_{y \in \Gamma}$ such that $B \supseteq \bigoplus_{\gamma \in \Gamma} K_{\gamma}$, then each $\Gamma_{n}$ is finite if and only if the socle $S$ of $B$ is dense. Clearly $S=\bigoplus_{\gamma \in \Gamma} K_{\gamma}$.

Necessity. Let $x=\left(x_{\gamma}\right) \in B$, and let $\left(z_{n}\right) \in \prod_{n \geqq 0} F_{n}$ and $m \geqq 0$ be such that for all $n>m, \sigma_{\gamma}\left(z_{n}\right)=x_{\gamma}$ for all $\gamma \in \Gamma_{n}$. For each $k \geqq 0$ let $e_{k}$ be the idempotent of $B$ whose $\gamma$ th component is 1 or 0 according as $\gamma \in \Gamma_{k}$ or $\gamma \notin \Gamma_{k}$. Then for each $q>m$, $\sum_{k=0}^{q} x e_{k} \in S$ as $\Gamma_{0}, \ldots, \Gamma_{q}$ are finite, and $x-\sum_{k=0}^{q} x e_{k} \in V\left(1-\sum_{k=0}^{q} e_{k}\right)$. Therefore as $\left(V\left(1-\sum_{k=0}^{q} e_{k}\right)\right)_{q \geqq 0}$ is a fundamental system of neighborhoods of zero, $S$ is dense in $B$.

Sufficiency. Let $n \geqq 0$. Then there exists $y=\left(y_{y}\right) \in S$ such that

$$
e_{n}-y \in V\left(1-\sum_{k=0}^{n} e_{k}\right) \text {. }
$$

Consequently $y_{\gamma}=1$ for all $\gamma \in \Gamma_{n}$, so $\Gamma_{n}$ is finite as $S=\bigoplus_{\gamma \in \Gamma} K_{\gamma}$.

THEOREM 24. Let $K$ be an infinite subfield of the algebraic closure of $Z_{p}$, equipped with the discrete topology. Let $A$ be a commutative semisimple indiscrete straight 
locally compact $K$-algebra, and let $S$ be the socle of $A$. Then $A$ is the topological direct product of a discrete commutative semisimple $K$-algebra and a $K$-algebra topologically isomorphic to the full locally compact algebra $B^{\prime}$ defined by a family of $K$-ingredients $\left(K_{\gamma}\right)_{\gamma \in \Gamma},\left(\Gamma_{n}\right)_{n \geqq 0},\left(F_{n}\right)_{n \geqq 0},\left(\sigma_{\gamma}\right)_{\gamma \in \Gamma}$ where each $\Gamma_{n}$ is finite if and only if the closure $S^{-}$of $S$ is an open ideal.

Proof. Necessity. Let $A$ be the topological direct product of an ideal $B$ topologically isomorphic to $B^{\prime}$ and a discrete ideal $C$. As $B$ has an identity element, every ideal of $B$ is an ideal of $A$, so the socle of $B$ is $B \cap S$. By Theorem 23, $(B \cap S)^{-}=B$. As $C$ and hence also $A / B$ are discrete, $B$ is open; therefore $(B \cap S)^{-}$ and hence also $S^{-}$are neighborhoods of zero, so $S^{-}$is an open ideal.

Sufficiency. As $S^{-}$is an open ideal of $A, S^{-}$is semisimple, indiscrete, and locally compact. By Theorem $20, S^{-}$is the topological direct product of a discrete ideal $C_{1}$ and an indiscrete ideal $B$ containing a compact open subring $V$ that possesses the identity element $e$ of $B$. As $C_{1}$ and hence $S^{-} / B$ are discrete, $B$ is open in $S^{-}$ and hence in $A$. As in the proof of Theorem 20,A is the topological direct product of $B$ and $C=\{x-x e: x \in A\}$, and $C$ is discrete. By Theorem 23, it suffices to show that the socle $S_{0}$ of $B$ is dense in $B$. As $B$ has an identity element, every ideal of $B$ is an ideal of $A$, so $S_{0}=S \cap B$. As $B$ is open, $B=S^{-} \cap B \subseteq(S \cap B)^{-}=S_{0}^{-}$. Thus $S_{0}$ is dense in $B$.

Definition. A commutative topological $K$-algebra $A$ is strongly semisimple if for every nonzero $x \in A$ there is a continuous homomorphism $u$ from $A$ into the topological $K$-algebra $K$ such that $u(x) \neq 0$.

THEOREM 25. Let $K$ be an infinite subfield of the algebraic closure of $Z_{p}$, equipped with the discrete topology. Let $B$ be an indiscrete straight locally compact $K$-algebra that contains a compact open subring $V$ such that the ideal generated by $V$ is $B$ itself, and let $S$ be the socle of $B$. Then $B$ is commutative and strongly semisimple if and only if $B$ is topologically isomorphic to a locally compact $K$-algebra $B^{\prime}$ defined by a family of $K$-ingredients $\left(K_{\gamma}\right)_{\gamma \in \Gamma},\left(\Gamma_{n}\right)_{n \geqq 0},\left(F_{n}\right)_{n \geqq 0},\left(\sigma_{\gamma}\right)_{\gamma \in \Gamma}$ such that $K_{\gamma}=K$ for all $\gamma \in \Gamma$. Moreover, the family of $K$-ingredients may be so chosen that $B^{\prime} \supseteq \bigoplus_{\gamma \in \Gamma} K_{\gamma}$ if and only if the annihilator of $S$ is $\{0\}$, and it may be so chosen that each $\Gamma_{n}$ is finite if and only if $S$ is dense.

Proof. Necessity. The assertion follows from Theorem 20, for we may take as our family $\left(\mathfrak{m}_{y}\right)_{y \in \Gamma}$ of closed regular maximal ideals those that are the kernels of nonzero continuous homomorphisms from $E$ onto $K$. Moreover, if the annihilator of $S$ is $\{0\}$, we need only consider the family of kernels of continuous homomorphisms from $E$ onto $K$ that take some minimal idempotent into 1 .

Sufficiency. As each $K_{y}=K$, the projections are clearly continuous homomorphisms from $B^{\prime}$ onto $K$, and the intersection of their kernels is $\{0\}$.

THEOREM 26. Let $K$ be an infinite subfield of the algebraic closure of $Z_{p}$, equipped with the discrete topology. Let $A$ be a commutative strongly semisimple indiscrete 
straight locally compact $K$-algebra, and let $S$ be the socle of $A$. Then $A$ is the topological direct product of a discrete commutative strongly semisimple $K$-algebra and $a$ $K$-algebra topologically isomorphic to a locally compact algebra $B^{\prime}$ defined by a family of K-ingredients $\left(K_{\gamma}\right)_{\gamma \in \Gamma},\left(\Gamma_{n}\right)_{n \geqq 0},\left(F_{n}\right)_{n \geqq 0},\left(\sigma_{\gamma}\right)_{\gamma \in \Gamma}$ such that $K_{\gamma}=K$ for all $\gamma \in \Gamma$. Moreover, the family may be so chosen that $B^{\prime} \supseteq \bigoplus_{\gamma \in \Gamma} K_{\gamma}$ if and only if the annihilator of the annihilator of $S$ is open, and it may be so chosen that each $\Gamma_{n}$ is finite if and only if $S^{-}$is open.

The proof is similar to those of Theorems 20, 22, and 24.

We shall next characterize those families of $K$-ingredients that determine compactly generated algebras.

LEMMA 9. Let $K$ be an infinite discrete absolutely algebraic field of prime characteristic. If $A$ is a commutative, straight, locally compact, compactly generated $K$-algebra, then $A$ contains a compact open subring $W$ that generates the vector space $A$.

Proof. By [10, Lemma 4], $A$ contains a compact open subring $V$; let $F$ be the subspace generated by $V$. Then $F$ is a subalgebra of finite codimension by Theorem 12. Assume that $F \neq A$. By induction it suffices to show that there is a compact open subring $W$ that generates a subspace properly containing $F$. Let $a \notin F$. Since $A / F$ is finite-dimensional, there exist $\lambda_{1}, \ldots, \lambda_{m} \in K$ such that $\lambda_{m} \neq 0$ and $\sum_{i=1}^{m} \lambda_{i}\left(a^{i}+F\right)=F$. Therefore $\sum_{i=1}^{m} \lambda_{i} a^{i} \in F$, so there exist $\beta_{1}, \ldots, \beta_{n} \in K$ and $v_{1}, \ldots, v_{n} \in V$ such that

$$
\sum_{i=1}^{m} \lambda_{i} a^{i}=\sum_{k=1}^{n} \beta_{k} v_{k}
$$

Let $L$ be the (finite) subfield of $K$ generated by $\lambda_{1}, \ldots, \lambda_{m}, \beta_{1}, \ldots, \beta_{n}$. Then $V^{\prime}=\sum_{\alpha \in L} \alpha . V$ is a compact open subring containing $V$, and $L . V^{\prime} \subseteq V^{\prime}$. Consequently, $W=V^{\prime}+V^{\prime} a+\cdots+V^{\prime} a^{m}+L . a+\cdots+L . a^{m}$ is a compact open subgroup of $A$; moreover, $V^{\prime} W \subseteq W$ and $L . W \subseteq W$. To prove that $W$ is a subring, therefore, it suffices to prove that $a^{n} \in W$ for all $n \geqq 1$. Assume that $a^{k} \in W$ for all $k<n$, where $n>m$. Let $w=\sum_{i=1}^{m} \lambda_{i} a^{i}$. Then as $w=\sum_{k=1}^{n} \beta_{k} v_{k}, w \in V^{\prime}$. Therefore

so

$$
\sum_{i=1}^{m} \lambda_{i} a^{i+n-m}=w a^{n-m}
$$

$$
a^{n}=\lambda_{m}^{-1} w a^{n-m}-\lambda_{m}^{-1} \sum_{i=1}^{m-1} \lambda_{i} a^{i+n-m} \in L . V^{\prime} W+L . W \subseteq W .
$$

Let $K$ be a subfield of the algebraic closure $\Omega$ of $Z_{p}$. For each $m \geqq 0$ we shall denote by $\tau_{m}$ the automorphism $x \rightarrow x^{p^{m}}$ of $\Omega$, and for each $f=\sum_{k=0}^{s} \alpha_{k} X^{k} \in K[X]$ we shall denote by $f_{m}$ the polynomial $\sum_{k=0}^{s} \tau_{m}\left(\alpha_{k}\right) X^{k}$. Clearly $f_{m} \in K[X]$.

LEMMA 10. Let $K$ be a subfield and $F$ a finite subfield of the algebraic closure $\Omega$ of $Z_{p}$, let $m \geqq 0$, let $L$ be the intersection of $K \cap F$ with the fixed field of $\tau_{m}$, and let 
$c$ be a generator of the multiplicative group $F^{*}$. Then $K$ and $F$ are linearly disjoint over $L$ if and only if for every $f \in K[X]$, if $f\left(\tau_{m}(c)\right)=0$, then $f(c)=0$.

Proof. Necessity. Suppose that there exists $f \in K[X]$ such that $f\left(\tau_{m}(c)\right)=0$ and $f(c) \neq 0$. The minimal polynomial $f_{1}$ of $\tau_{m}(c)$ over $K$ then satisfies $f_{1}\left(\tau_{m}(c)\right)=0$, $f_{1}(c) \neq 0$, so we may assume that $f=f_{1}$. By hypothesis, $[F: L]=[K(F): K]$ [1, Proposition 4, p. 79]. But $\tau_{m}(c)$ is a generator of $F^{*}$, so $K(F)=K\left(\tau_{m}(c)\right)$ and $F=L(c)$. Thus as $[L(c): L]=\left[K\left(\tau_{m}(c)\right): K\right]$, the minimal polynomial $g$ of $c$ over $L$ has the same degree as $f$. Let $g=\sum_{k=0}^{s} \alpha_{k} X^{k} \in L[X]$. Then

$$
\begin{aligned}
g\left(\tau_{m}(c)\right) & =\sum_{k=0}^{s} \alpha_{k} \tau_{m}(c)^{k}=\sum_{k=0}^{s} \tau_{m}\left(\alpha_{k}\right) \tau_{m}(c)^{k} \\
& =\tau_{m}\left(\sum_{k=0}^{s} \alpha_{k} c^{k}\right)=\tau_{m}(0)=0 .
\end{aligned}
$$

Hence as $g \in K[X], g=f$, so $f(c)=g(c)=0$, a contradiction.

Sufficiency. Let $g=\sum_{k=0}^{r} \beta_{k} X^{k}$ be the minimal polynomial of $c$ over $K$, and let $f$ be the minimal polynomial of $\tau_{m}(c)$ over $K$. Now

$$
g_{m}\left(\tau_{m}(c)\right)=\tau_{m}(g(c))=\tau_{m}(0)=0
$$

so by hypothesis $g_{m}(c)=0$. As $\operatorname{deg} g_{m}=\operatorname{deg} g$, therefore $g_{m}=g$. Consequently, the coefficients of $g$ belong to the fixed field of $\tau_{m}$. Moreover, $c$ is a primitive $q$ th root of unity where $q+1$ is the order of $F$, so $g$ divides the cyclotomic polynomial of index $q$; therefore every root of $g$ in $\Omega$ is a power of $c$, and consequently the coefficients of $g$ belong to $Z_{p}(c)=F$. Hence $g \in L[X]$, and thus $g$ is the minimal polynomial of $c$ over $L$. As $F=L(c)$ and $K(F)=K(c)$,

$$
[F: L]=\operatorname{deg}_{L} c=r=\operatorname{deg}_{K} c=[K(F): K],
$$

so $F$ and $K$ are linearly disjoint over $L$ [1, Proposition 4, p. 79].

LEMMA 11. Let $K$ be a subfield and $F$ a finite subfield of the algebraic closure $\Omega$ of $Z_{p}$, let $\left(\sigma_{k}\right)_{1 \leqq k \leqq n}$ be a sequence of distinct automorphisms of $F$, let $A$ be the $K$-algebra $K(F)^{n}$, the cartesian product of $n$ copies of $K(F)$, and let $V=\left\{\left(\sigma_{1}(x), \ldots\right.\right.$, $\left.\left.\sigma_{n}(x)\right): x \in F\right\}$, a subfield of $A$ isomorphic to $F$. The subspace $B$ of $A$ generated by $V$ is a subalgebra, and $B=A$ if and only if for all distinct $i, j \in[1, n], K$ and $F$ are not linearly disjoint over the intersection of $K$ with the fixed field of $\sigma_{i}^{-1} \sigma_{j}$.

Proof. As $V$ is a subfield of $A, B$ is clearly closed under multiplication and hence is a subalgebra. Let $c$ be a generator of the multiplicative group $F^{*}$. Now each $\sigma_{k}$ is the restriction to $F$ of $\tau_{m_{k}}$ for some $m_{k} \geqq 0$ [1, Proposition 5, p. 170]. By Lemma 10, the condition is therefore equivalent to the assertion that if $i \neq j$, there exists $f \in K[X]$ such that $f\left(\sigma_{i}^{-1}\left(\sigma_{j}(c)\right)\right)=0$ and $f(c) \neq 0$. If $f=\sum_{k=0}^{s} \alpha_{k} X^{k} \in K[X]$, if $a \in F$ and if $m \geqq 0$, then for any automorphism $\sigma$ of $F$,

$$
f_{m}(\sigma(a))=\tau_{m}\left(\sum_{k=0}^{s} \alpha_{k} \tau_{m}^{-1}(\sigma(a))^{k}\right)=\tau_{m}\left(f\left(\tau_{m}^{-1}(\sigma(a))\right)\right) .
$$


Consequently, our condition is equivalent to the following: $\left(^{*}\right)$ If $i \neq j$, there exists $g \in K[X]$ such that $g\left(\sigma_{i}(c)\right) \neq 0$ and $g\left(\sigma_{j}(c)\right)=0$. Clearly

$$
B=\left\{\left(g\left(\sigma_{1}(c)\right), \ldots, g\left(\sigma_{n}(c)\right)\right): g \in K[X]\right\}
$$

as every nonzero element of $F$ is a power of $c$. Hence if $B=A$, then $\left(^{*}\right)$ holds.

It remains for us to show that $\left(^{*}\right)$ implies that $B=A$. By $\left({ }^{*}\right)$, if $i \neq j$, there exists $a_{i j} \in B$ whose $i$ th component is not zero but whose $j$ th component is zero. Hence $a_{i}=\prod_{j \neq i} a_{i j}$ is an element of $B$ whose $i$ th component $\alpha_{i}$ is its only nonzero component. As $\alpha_{i}$ is algebraic over $K$ and as $K(F)=K\left(\sigma_{i}(c)\right)$, there exist $\lambda_{0}, \ldots, \lambda_{s} \in K$ such that $\alpha_{i}^{-1}=\sum_{k=0}^{s} \lambda_{k} \sigma_{i}(c)^{k}$, whence

$$
e_{i}=(0, \ldots, 0,1,0, \ldots, 0)=a_{i} \sum_{k=0}^{s} \lambda_{k}\left(\sigma_{1}(c), \ldots, \sigma_{n}(c)\right)^{k} \in B .
$$

Consequently if $\beta \in K(F)$, there exist $\mu_{0}, \ldots, \mu_{r} \in K$ such that $\beta=\sum_{k=0}^{r} \mu_{k} \sigma_{i}(c)^{k}$, whence

$$
(0, \ldots, 0, \beta, 0, \ldots, 0)=e_{i} \sum_{k=0}^{r} \mu_{k}\left(\sigma_{1}(c), \ldots, \sigma_{n}(c)\right)^{k} \in B .
$$

Therefore $B=A$.

THEOREM 27. Let $K$ be an infinite subfield of the algebraic closure of $Z_{p}$, equipped with the discrete topology. If $A$ is a commutative semisimple indiscrete straight locally compact $K$-algebra, then $A$ is compactly generated if and only if $A$ is topologically isomorphic to the full locally compact algebra defined by a family of $K$-ingredients $\left(K_{\gamma}\right)_{y \in \Gamma},\left(\Gamma_{n}\right)_{n \geqq 0},\left(F_{n}\right)_{n \geqq 0},\left(\sigma_{\gamma}\right)_{\gamma \in \Gamma}$ satisfying the following conditions:

$1^{\circ}$. For each $n \geqq 0, \gamma \rightarrow \sigma_{\gamma}$ is an injection from $\Gamma_{n}$ into Aut $F_{n}$; hence $\Gamma_{n}$ is finite for each $n \geqq 0$.

$2^{\circ}$. For each $n \geqq 0, K_{y}=K\left(F_{n}\right)$ for all $\gamma \in \Gamma_{n}$.

$3^{\circ}$. For each $n \geqq 0$, if $\beta$ and $\gamma$ are distinct members of $\Gamma_{n}$, then $K$ and $F_{n}$ are not linearly disjoint over the intersection of $K$ with the fixed field of $\sigma_{\beta}^{-1} \sigma_{\gamma}$.

Proof. First, assume that $A$ is a commutative, semisimple, indiscrete, straight, locally compact, compactly generated $K$-algebra. By Lemma $9, A$ contains a compact open subring $V$ that generates the vector space $A$. In particular, $A$ is the ideal generated by $V$, so by Theorem 19 we may assume that $A$ is a locally compact algebra defined by a family of $K$-ingredients $\left(K_{\gamma}\right)_{\gamma \in \Gamma},\left(\Gamma_{n}\right)_{n \geqq 0},\left(F_{n}\right)_{n \geqq 0},\left(\sigma_{\gamma}\right)_{\gamma \in \Gamma}$ and that the ring $V$ defined by this family generates $A$. If there were distinct $\alpha, \beta \in \Gamma_{n}$ such that $\sigma_{\alpha}=\sigma_{\beta}$, then $x_{\alpha}=x_{\beta}$ for all $\left(x_{\gamma}\right) \in V$ and hence also for each $\left(x_{\gamma}\right) \in A$, the vector space generated by $V$, in contradiction to the definition of an algebra defined by a family of $K$-ingredients. Consequently, $1^{\circ}$ holds. Moreover, as $A$ is the vector space generated by $V, K_{\gamma}$ is clearly $K\left(F_{n}\right)$ for all $\gamma \in \Gamma_{n}$.

To complete the proof, therefore, it suffices to show that if $1^{\circ}$ and $2^{\circ}$ hold, then $3^{\circ}$ holds if and only if $V$ generates $A$, where $V$ is the ring and $A$ the full algebra defined by this family. For each $n \geqq 0$ let $e_{n}$ be the idempotent whose $\gamma$ th component 
is 1 or 0 according as $\gamma \in \Gamma_{n}$ or $\gamma \notin \Gamma_{n}$. By the definition of $A$, for each $a \in A$ there exists $m \geqq 0$ such that $a$ is the sum of elements belonging to $A e_{0}, \ldots, A e_{m}$, and $V$. Consequently as $A e_{n} \cap V=V e_{n}, V$ generates $A$ if and only if $V e_{n}$ generates $A e_{n}$ for all $n \geqq 0$. But since $A e_{n}$ is canonically isomorphic to the cartesian product of a finite number of copies of the $K$-algebra $K\left(F_{n}\right), V e_{n}$ generates $A e_{n}$ for all $n \geqq 0$ if and only if $3^{\circ}$ holds by Lemma 11 .

COROLlaRY. An indiscrete straight locally compact $K$-algebra $A$ is commutative, strongly semisimple, and compactly generated if and only if $A$ is topologically isomorphic to the full locally compact algebra defined by a family of $K$-ingredients $\left(K_{\gamma}\right)_{y \in \Gamma},\left(\Gamma_{n}\right)_{n \geqq 0},\left(F_{n}\right)_{n \geqq 0},\left(\sigma_{\gamma}\right)_{y \in \Gamma}$ satisfying the following conditions:

$1^{\circ}$. For each $n \geqq 0, \gamma \rightarrow \sigma_{\gamma}$ is an injection from $\Gamma_{n}$ into Aut $F_{n}$.

$2^{\circ}$. $K_{\gamma}=K$ for all $\gamma \in \Gamma$.

Proof. If $B \supseteq \bigoplus_{\gamma \in \Gamma} K_{\gamma}$, then for each $\gamma \in \Gamma, B$ contains a subfield isomorphic to $K_{\gamma}$, so $B$ is strongly semisimple if and only if $K_{\gamma}=K$ for all $\gamma \in \Gamma$. The assertion therefore follows from Theorem 27.

In general, we cannot expect too close a relationship between two families of $K$-ingredients defining isomorphic locally compact algebras. Indeed, if $B$ is a locally compact algebra defined by $\left(K_{\gamma}\right)_{y \in \Gamma},\left(\Gamma_{n}\right)_{n \geqq 0},\left(F_{n}\right)_{n \geqq 0},\left(\sigma_{\gamma}\right)_{y \in \Gamma}$ and if $B$ does not contain $\bigoplus_{y \in \Gamma} K_{y}$, then by Lemma $7 \Gamma$ may contain a subset $\Gamma^{\prime}$ whose complement is infinite such that $B$ is isomorphic to a locally compact algebra defined by a family of $K$-ingredients whose family of field extensions of $K$ is indexed by $\Gamma^{\prime}$. Consequently, we shall confine our attention to algebras defined by a family of $K$-ingredients that contain the outer direct sum of the family of field extensions that is part of the given family of $K$-ingredients.

Definition. Let $K$ be an infinite subfield of the algebraic closure $\Omega$ of $Z_{p}$. An equivalence of a family of $K$-ingredients $\left(K_{\gamma}\right)_{\gamma \in \Gamma},\left(\Gamma_{n}\right)_{n \geqq 0},\left(F_{n}\right)_{n \geqq 0},\left(\sigma_{\gamma}\right)_{y \in \Gamma}$ with a family of $K$-ingredients $\left(L_{\delta}\right)_{\delta \in \Delta},\left(\Delta_{n}\right)_{n \geqq 0},\left(H_{n}\right)_{n \geqq 0},\left(\tau_{\delta}\right)_{\delta \in \Delta}$ is a quadruple $\left(\phi,\left(h_{\delta}\right)_{\delta \in \Delta}, \psi: M^{\prime} \rightarrow M,\left(\rho_{s}\right)_{s \in M^{\prime}}\right)$ satisfying the following conditions:

$1^{\circ}$. $\phi$ is a bijection from $\Delta$ onto $\Gamma$.

$2^{\circ}$. For all $\delta \in \Delta, h_{\delta}$ is a $K$-isomorphism from $K_{\phi(\delta)}$ onto $L_{\delta}$.

$3^{\circ}$. $M$ and $M^{\prime}$ are subsets of the natural numbers $N$ whose complements are finite.

$4^{\circ} . \psi$ is a bijection from $M^{\prime}$ onto $M$ such that $\Gamma_{\psi(s)}=\phi\left(\Delta_{s}\right)$ and $F_{\psi(s)}=H_{s}$ for all $s \in M^{\prime}$.

$5^{\circ}$. For each $s \in M^{\prime}, \rho_{s}$ is an automorphism of $F_{\psi(s)}$ satisfying

$$
\tau_{\delta} \circ \rho_{s}=h_{\delta} \circ \sigma_{\phi(\delta)}
$$

for all $\delta \in \Delta_{s}$.

Two families of $K$-ingredients are equivalent if there is an equivalence between them. 
THEOREM 28. Let $K$ be an infinite subfield of the algebraic closure $\Omega$ of $Z_{p}$. Let $B$ be a locally compact algebra and $V$ the ring defined by a family of $K$-ingredients $\left(K_{\gamma}\right)_{\gamma \in \Gamma},\left(\Gamma_{n}\right)_{n \geqq 0},\left(F_{n}\right)_{n \geqq 0},\left(\sigma_{\gamma}\right)_{\gamma \in \Gamma}$ such that $B \supseteq \bigoplus_{\gamma \in \Gamma} K_{\gamma}$, and let $B^{\prime}$ be a locally compact algebra and $V^{\prime}$ the ring defined by a family of $K$-ingredients $\left(L_{\delta}\right)_{\delta \in \Delta}$, $\left(\Delta_{n}\right)_{n \geqq 0},\left(H_{n}\right)_{n \geqq 0},\left(\tau_{\delta}\right)_{\delta \in \Delta}$ such that $B^{\prime} \supseteq \bigoplus_{\delta \in \Delta} L_{\delta}$. If $h$ is a topological isomorphism from $B$ onto $B^{\prime}$, then there is an equivalence $\left(\phi,\left(h_{\delta}\right)_{\delta \in \Delta}, \psi: M^{\prime} \rightarrow M,\left(\rho_{s}\right)_{s \in M^{\prime}}\right)$ of these two families of $K$-ingredients such that

$$
h\left(\left(x_{\gamma}\right)\right)=\left(h_{\delta}\left(x_{\phi(\delta)}\right)\right)
$$

for all $\left(x_{\gamma}\right) \in B$. Conversely, if $\left(\phi,\left(h_{\delta}\right)_{\delta \in \Delta}, \psi: M^{\prime} \rightarrow M,\left(\rho_{s}\right)_{s \in M^{\prime}}\right)$ is an equivalence of these two families of K-ingredients, then $h:\left(x_{\gamma}\right) \rightarrow\left(h_{\delta}\left(x_{\phi(\delta)}\right)\right)$ is a topological isomorphism from $B$ onto a locally compact algebra defined by $\left(L_{\delta}\right)_{\delta \in \Delta},\left(\Delta_{n}\right)_{n \geqq 0}$, $\left(H_{n}\right)_{n \geqq 0},\left(\tau_{\delta}\right)_{\delta \in \Delta}$ that contains $\bigoplus_{\delta \in \Delta} L_{\delta}$.

Proof. For each $\gamma \in \Gamma$, let $e_{\gamma}$ be the idempotent of $B$ whose $\gamma$ th component is 1 and whose remaining components are 0 ; similarly, we define $e_{\delta}^{\prime} \in B^{\prime}$ for each $\delta \in \Delta$. For each $i \geqq 0$, let $e_{i} \in V$ be the idempotent whose $\gamma$ th component is 1 or 0 according as $\gamma \in \Gamma_{i}$ or $\gamma \notin \Gamma_{i}$; similarly we define $e_{i}^{\prime} \in V^{\prime}$. The minimal idempotents of $B$ are clearly the idempotents $e_{\gamma}$ where $\gamma \in \Gamma$, and the minimal idempotents of $B^{\prime}$ are similarly the idempotents $e_{\delta}^{\prime}$ where $\delta \in \Delta$. Hence as $h$ is an algebraic isomorphism, there is a bijection $\phi$ from $\Delta$ onto $\Gamma$ such that $h\left(e_{\phi(\delta)}\right)=e_{\delta}^{\prime}$ for all $\delta \in \Delta$. Then for each $\delta \in \Delta, h\left(B e_{\phi(\delta)}\right)=B^{\prime} e_{\delta}^{\prime}$, so

$$
h_{\delta}=\operatorname{pr}_{\delta} \circ h \circ \operatorname{in}_{\phi(\delta)}
$$

is a $K$-isomorphism from $K_{\phi(\delta)}$ onto $L_{\delta}$, where in $_{\phi(\delta)}$ is the canonical injection from $K_{\phi(\delta)}$ into $B, \mathrm{pr}_{\delta}$ the canonical projection from $B^{\prime}$ onto $L_{\delta}$, since $\mathrm{in}_{\phi(\delta)}$ is actually a $K$-isomorphism from $K_{\phi(\delta)}$ onto $B e_{\phi(\delta)}$ and $\mathrm{pr}_{\delta}$ is a $K$-isomorphism from $B^{\prime} e_{\delta}^{\prime}$ onto $L_{\delta}$.

We shall next prove that for each $\left(x_{y}\right) \in B$,

$$
h\left(\left(x_{\gamma}\right)\right)=\left(h_{\delta}\left(x_{\phi(\delta)}\right)\right) .
$$

Let $x=\left(x_{\gamma}\right)$, and let $\left(y_{\delta}\right)=h(x)$. For each $\delta \in \Delta$,

and also

$$
\begin{aligned}
\operatorname{pr}_{\delta}\left(h\left(e_{\phi(\delta)} x\right)\right) & =\operatorname{pr}_{\delta}\left(h\left(\operatorname{in}_{\phi(\delta)}\left(x_{\phi(\delta)}\right)\right)\right) \\
& =h_{\delta}\left(x_{\phi(\delta)}\right),
\end{aligned}
$$

$$
\operatorname{pr}_{\delta}\left(h\left(e_{\phi(\delta)} x\right)\right)=\operatorname{pr}_{\delta}\left(h\left(e_{\phi(\delta)}\right) h(x)\right)=\operatorname{pr}_{\delta}\left(e_{\delta}^{\prime}\right) \cdot \operatorname{pr}_{\delta}(h(x))=1 \cdot y_{\delta} .
$$

Thus $h\left(\left(x_{\gamma}\right)\right)=\left(h_{\delta}\left(x_{\phi(\delta)}\right)\right)$.

We observe next that $h\left(e_{i}\right)$ is the idempotent of $B^{\prime}$ whose $\delta$ th component is 1 or 0 according as $\delta \in \phi^{-1}\left(\Gamma_{i}\right)$ or $\delta \notin \phi^{-1}\left(\Gamma_{i}\right)$. Indeed, $h\left(e_{i}\right)$ is an idempotent of $B^{\prime}$, so there is a subset $\Delta_{i}^{\prime}$ of $\Delta$ such that the $\delta$ th component of $h\left(e_{i}\right)$ is 1 or 0 according as $\delta \in \Delta_{i}^{\prime}$ or $\delta \notin \Delta_{i}^{\prime}$. Thus $\delta \in \Delta_{i}^{\prime}$ if and only if $h\left(e_{i}\right) e_{\delta}^{\prime}=e_{\delta}^{\prime}$, or equivalently, if 
and only if $e_{i} h^{-1}\left(e_{\delta}^{\prime}\right)=h^{-1}\left(e_{\delta}^{\prime}\right)$, that is, if and only if $e_{2} e_{\phi(\delta)}=e_{\phi(\delta)}$, or finally, if and only if $\phi(\delta) \in \Gamma_{i}$. Hence $\Delta_{i}^{\prime}=\phi^{-1}\left(\Gamma_{i}\right)$. Similarly, $h^{-1}\left(e_{i}^{\prime}\right)$ is the idempotent of $B$ whose $\gamma$ th component is 1 or 0 according as $\gamma \in \phi\left(\Delta_{i}\right)$ or $\gamma \notin \phi\left(\Delta_{i}\right)$.

For each $i \geqq 0$ let

$$
J_{i}=\left\{k \geqq 0: \phi^{-1}\left(\Gamma_{k}\right) \cap \Delta_{i} \neq \varnothing\right\}, \quad J_{i}^{\prime}=\left\{k \geqq 0: \phi\left(\Delta_{k}\right) \cap \Gamma_{i} \neq \varnothing\right\} .
$$

Suppose that $J_{i}$ were infinite. Then by the preceding there would exist a strictly increasing sequence of integers $\left(k_{r}\right)_{r} \geqq 0$ such that $h\left(e_{k_{r}}\right) e_{i}^{\prime} \neq 0$ for all $r \geqq 0$. Let $f_{r}^{\prime}=h\left(e_{k_{r}}\right) e_{i}^{\prime}$. If $r \neq s$, then $f_{r}^{\prime} f_{s}^{\prime}=h\left(e_{k_{r}} e_{k_{s}}\right) e_{i}^{\prime}=0$, so $\left(f_{r}^{\prime}\right)_{r \geqq 0}$ would be a sequence of distinct idempotents in $B^{\prime} e_{i}^{\prime}$, which is discrete as $B^{\prime} e_{i}^{\prime} \cap V^{\prime}\left(1-\sum_{k=0}^{i} e_{k}^{\prime}\right)=\{0\}$. But as $\left(V\left(1-\sum_{k=0}^{m} e_{k}\right)\right)_{m \geqq 0}$ is a fundamental system of neighborhoods of zero in $B$, $e_{k_{r}} \rightarrow 0$, so $f_{r}^{\prime}=h\left(e_{k_{r}}\right) e_{i}^{\prime} \rightarrow 0$, a contradiction. Therefore $J_{i}$ is finite, and similarly $J_{i}^{\prime}$ is finite.

As $h$ is a topological isomorphism, there exists $m \geqq 0$ such that $h\left(V\left(1-\sum_{i=0}^{m} e_{i}\right)\right)$ $\subseteq V^{\prime}$ and $V^{\prime}\left(1-\sum_{i=0}^{m} e_{i}^{\prime}\right) \subseteq h(V)$. Let

$$
M=N-\left([0, m] \cup J_{0} \cup \cdots \cup J_{m}\right), \quad M^{\prime}=N-\left([0, m] \cup J_{0}^{\prime} \cup \cdots \cup J_{m}^{\prime}\right) .
$$

We shall prove that for each $r \in M$ there is a unique $s \in M^{\prime}$ such that $\Gamma_{r}=\phi\left(\Delta_{s}\right)$. Indeed, let $r \in M$. Then there exists $s \geqq 0$ such that $\Delta_{s} \cap \phi^{-1}\left(\Gamma_{r}\right) \neq \varnothing$. Consequently $r \in J_{s}$, so $s>m$, and thus $e_{s}^{\prime} \in V^{\prime}\left(1-\sum_{i=0}^{m} e_{i}^{\prime}\right) \subseteq h(V)$. Therefore as $h^{-1}\left(e_{s}^{\prime}\right) \in V$ and as $\phi\left(\Delta_{s}\right) \cap \Gamma_{r}=\phi\left(\Delta_{s} \cap \phi^{-1}\left(\Gamma_{r}\right)\right) \neq \varnothing, \Gamma_{r} \subseteq \phi\left(\Delta_{s}\right)$ by the preceding discussion of the idempotents $h^{-1}\left(e_{i}^{\prime}\right)$ and the definition of $V$. As $r>m, e_{r} \in V\left(1-\sum_{i=0}^{m} e_{i}\right)$, so $h\left(e_{r}\right) \in V^{\prime}$. Therefore as $\Delta_{s} \cap \phi^{-1}\left(\Gamma_{r}\right) \neq \varnothing, \Delta_{s} \subseteq \phi^{-1}\left(\Gamma_{r}\right)$ by the preceding discussion of the idempotents $h\left(e_{i}\right)$ and the definition of $V^{\prime}$. Hence $\Gamma_{r}=\phi\left(\Delta_{s}\right)$; in particular, $\phi\left(\Delta_{s}\right) \cap \Gamma_{i}=\varnothing$ if $i \neq r$ and hence if $i \leqq m$, so $s \notin J_{0}^{\prime} \cup \cdots \cup J_{m}^{\prime}$. Thus $s \in M^{\prime}$. Clearly $s$ is unique. Similarly, for each $s \in M^{\prime}$ there is a unique $r \in M$ such that $\Delta_{s}=\phi^{-1}\left(\Gamma_{r}\right)$. Hence there is a bijection $\psi$ from $M^{\prime}$ onto $M$ such that $\Gamma_{\psi(s)}=\phi\left(\Delta_{s}\right)$ for all $s \in M^{\prime}$.

Let $s \in M^{\prime}$. Then $h\left(B e_{\psi(s)}\right)=B^{\prime} h\left(e_{\psi(s)}\right)=B^{\prime} e_{s}^{\prime}$ since $\phi^{-1}\left(\Gamma_{\psi(s)}\right)=\Delta_{s}$. As $\psi(s)>m$, $V e_{\psi(s)}=V e_{\psi(s)}\left(1-\sum_{i=0}^{m} e_{i}\right) \subseteq V\left(1-\sum_{i=0}^{m} e_{i}\right)$, whence

$$
h\left(V e_{\psi(s)}\right) \subseteq V^{\prime} \cap B^{\prime} e_{s}^{\prime}=V^{\prime} e_{s}^{\prime} .
$$

Similarly, as $s>m, h^{-1}\left(V^{\prime} e_{s}^{\prime}\right) \subseteq V e_{\psi(s)}$. Therefore $h\left(V e_{\psi(s)}\right)=V^{\prime} e_{s}^{\prime}$. To show that $F_{\psi(s)}=H_{s}$, let $\gamma \in \Gamma_{\psi(s)}$ and $\delta \in \Delta_{s}$. Then the restriction to $V e_{\psi(s)}$ of the canonical projection $\mathrm{pr}_{\gamma}$ from $B$ onto $K_{\gamma}$ is an isomorphism from $V e_{\psi(s)}$ onto $F_{\psi(s)}$, and similarly $\left(\operatorname{pr}_{\delta} \mid V^{\prime} e_{s}^{\prime}\right)$ is an isomorphism from $V^{\prime} e_{s}^{\prime}$ onto $H_{s}$. Hence as $h\left(V e_{\psi(s)}\right)$ $=V^{\prime} e_{s}^{\prime},\left(\operatorname{pr}_{\delta} \mid V^{\prime} e_{s}^{\prime}\right) \circ h \circ\left(\mathrm{pr}_{\gamma} \mid V e_{\psi(s)}\right)^{-1}$ is an isomorphism from $F_{\psi(s)}$ onto $H_{s}$. Thus $F_{\psi(s)}$ and $H_{s}$ are finite subfields of $\Omega$ having the same number of elements, so $F_{\psi(s)}=H_{s}$. To prove $5^{\circ}$ of the Definition, let $\delta_{s}$ be some member of $\Delta_{s}$. Then $\phi\left(\delta_{s}\right) \in \phi\left(\Delta_{s}\right)=\Gamma_{\psi(s)}$, so $h_{\delta}\left(F_{\psi(s)}\right)$ is a subfield of $L_{\delta}$ having the same number of elements as $F_{\psi(s)}=H_{s}$ and hence is identical with $H_{s}$. Therefore

$$
\rho_{s}=\tau_{\delta_{s}}^{-1} \circ h_{\delta_{s}} \circ \sigma_{\phi\left(\delta_{s}\right)}
$$


is an automorphism of $F_{\psi(s)}$. We shall prove that for any $\delta \in \Delta_{s}$,

$$
\tau_{\delta} \circ \rho_{s}=h_{\delta} \circ \sigma_{\phi(\delta)}
$$

Let $z \in F_{\psi(s)}$, and let $x_{\gamma}=\sigma_{\gamma}(z)$ for all $\gamma \in \Gamma_{\psi(s)}, x_{\gamma}=0$ for all $\gamma \notin \Gamma_{\psi(s)}$. Then $\left(x_{\gamma}\right) \in V e_{\psi(s)}$, so $h\left(\left(x_{\gamma}\right)\right) \in V^{\prime} e_{s}^{\prime}$, that is, $\left(h_{\delta}\left(x_{\phi(\delta)}\right)\right) \in V^{\prime} e_{s}^{\prime}$. Hence there exists $z^{\prime} \in H_{s}$ $=F_{\psi(s)}$ such that $h_{\delta}\left(x_{\phi(\delta)}\right)=\tau_{\delta}\left(z^{\prime}\right)$ for all $\delta \in \Delta_{s}$. In particular, $z^{\prime}=\tau_{\delta_{s}}^{-1}\left(h_{\delta_{s}}\left(x_{\phi\left(\delta_{s}\right)}\right)\right)$. Therefore

$$
\begin{aligned}
\left(\tau_{\delta} \circ \rho_{s}\right)(z) & =\tau_{\delta} \tau_{\delta_{s}}^{-1} h_{\delta_{s}} \sigma_{\phi\left(\delta_{s}\right)}(z)=\tau_{\delta} \tau_{\delta_{s}}^{-1} h_{\delta_{s}}\left(x_{\phi\left(\delta_{s}\right)}\right) \\
& =\tau_{\delta}\left(z^{\prime}\right)=h_{\delta}\left(x_{\phi(\delta)}\right)=h_{\delta}\left(\sigma_{\phi(\delta)}(z)\right) .
\end{aligned}
$$

The proof of the converse offers no difficulty.

\section{REFERENCES}

1. N. Bourbaki, Algèbre, Hermann, Paris, 1950, Chapters 4-5.

2. - Topologie générale, 3rd ed., Hermann, Paris, 1961, Chapters 1-2.

3. —_ Topologie générale, 3rd ed., Hermann, Paris, 1960, Chapters 3-4.

4. - Topologie générale, 2nd ed., Hermann, Paris, 1958, Chapter 9.

5. —_ Topologie générale, 2nd ed., Hermann, Paris, 1961, Chapter 10.

6. - Espaces vectoriels topologiques, Hermann, Paris, 1953, Chapters 1-2.

7. J. Braconnier, Sur les groupes topologiques localement compacts, J. Math. Pures Appl. 27 (1948), 1-86.

8. Nathan Jacobson, Structure of rings, Colloq. Publ., Vol. 37, Amer. Math. Soc., Providence, R. I., 1956.

9. Irving Kaplansky, Topological methods in valuation theory, Duke Math. J. 14 (1947), 527-541.

10. —_ Locally compact rings, Amer. J. Math. 70 (1948), 447-459.

11. John L. Kelley, General topology, Van Nostrand, Princeton, N. J., 1955.

12. Yosikazu Otobe, On locally compact fields, Japan. J. Math. 19 (1945), 189-202.

13. L. Pontrjagin, Topological groups, Princeton Univ. Press, Princeton, N. J., 1946.

14. Seth Warner, Modern algebra, Vol. I, Prentice-Hall, Englewood Cliffs, N. J., 1965.

15. —-, Modern algebra, Vol. II, Prentice-Hall, Englewood Cliffs, N. J., 1965.

16. - Locally compact simple rings having minimal left ideals, Trans. Amer. Math. Soc. 125 (1966), 395-405.

17. Leopoldo Nachbin, On strictly minimal topological division rings, Bull. Amer. Math. Soc. 55 (1949), 1128-1136.

DUKe UNIVERSITY, Durham, North Carolina 